\title{
Systems Pharmacology Dissecting Holistic Medicine for Treatment of Complex Diseases: An Example Using Cardiocerebrovascular Diseases Treated by TCM
}

\author{
Yonghua Wang, ${ }^{1}$ Chunli Zheng, ${ }^{2}$ Chao Huang, ${ }^{2}$ Yan $\mathrm{Li}^{3}{ }^{3}$ Xuetong Chen, ${ }^{2}$ Ziyin Wu, \\ Zhenzhong Wang, ${ }^{4}$ Wei Xiao, ${ }^{4}$ and Boli Zhang' \\ ${ }^{1}$ Evidence-Based Medicine Centre, Tianjin University of Traditional Chinese Medicine, The First Affiliated Hospital, \\ Tianjin University of Traditional Chinese Medicine, Tianjin 300193, China \\ ${ }^{2}$ Bioinformatics Center, College of Life Sciences, Northwest A\&F University, Yangling, Shaanxi 712100, China \\ ${ }^{3}$ School of Chemical Engineering, Dalian University of Technology, Dalian 116024, China \\ ${ }^{4}$ State Key Laboratory of New-Tech for Chinese Medicine Pharmaceutical Process, Lianyungang, Jiangsu 22001, China
}

Correspondence should be addressed to Yonghua Wang; yh_wang@nwsuaf.edu.cn and Boli Zhang; zhangbolipr@163.com

Received 10 February 2015; Revised 8 April 2015; Accepted 15 April 2015

Academic Editor: Yew-Min Tzeng

Copyright (c) 2015 Yonghua Wang et al. This is an open access article distributed under the Creative Commons Attribution License, which permits unrestricted use, distribution, and reproduction in any medium, provided the original work is properly cited.

\begin{abstract}
Holistic medicine is an interdisciplinary field of study that integrates all types of biological information (protein, small molecules, tissues, organs, external environmental signals, etc.) to lead to predictive and actionable models for health care and disease treatment. Despite the global and integrative character of this discipline, a comprehensive picture of holistic medicine for the treatment of complex diseases is still lacking. In this study, we develop a novel systems pharmacology approach to dissect holistic medicine in treating cardiocerebrovascular diseases (CCDs) by TCM (traditional Chinese medicine). Firstly, by applying the TCM active ingredients screened out by a systems-ADME process, we explored and experimentalized the signed drug-target interactions for revealing the pharmacological actions of drugs at a molecule level. Then, at a/an tissue/organ level, the drug therapeutic mechanisms were further investigated by a target-organ location method. Finally, a translational integrating pathway approach was applied to extract the diseases-therapeutic modules for understanding the complex disease and its therapy at systems level. For the first time, the feature of the drug-target-pathway-organ-cooperations for treatment of multiple organ diseases in holistic medicine was revealed, facilitating the development of novel treatment paradigm for complex diseases in the future.
\end{abstract}

\section{Introduction}

Holistic medicine is a system of health care which focuses on the whole person rather than just on the illness or part of the body that is not healthy $[1,2]$. The concept of holistic medicine can trace back to the 4 th century B.C. [3]. Since then, considerable knowledge has been accumulated concerning its efficacy and safety in treating ill health. However, from the beginning of the twentieth century, the principle of holistic medicine has been gradually falling out of favor in Western societies, with the enormous advancements in what we now call "allopathic" medicine. Paradoxically, the increasing evidence in clinic shows the allopathic medicine has got into trouble for handling complex diseases, particularly, the chronic diseases, as it mainly focused on particular body components to treat. In this case, the nonreductionist systems medicine [4], which draws on theories from holistic medicine, has been proposed to consider complex interactions within the human body to promote an individual's health in light of their genomics, behavior, and the external environment.

The complex diseases, such as tumors, diabetes, cardiocerebrovascular diseases (CCDs), infectious diseases, and many more, largely arise from an integration of genetic, environmental, and lifestyle factors [5]. A notable example is the cardiorenal syndrome (CRS) [6], which is employed 
for the coexistence of heart and kidney malfunction. And so far, there is still no effective cure in allopathic medicine to head off the process from cardiovascular diseases (CVDs) to kidney disease. A major obstacle to complex disease treatment is that the present one drug-one target-one disease philosophy is incapable of dealing with the complex nature of these illnesses. Thus, people are now returning to holistic medicine to find newfangled therapeutics aiming at multiple targets/organs interactions for preventing and treating of diseases.

There are some health-prevention systems that come under the umbrella of holistic medicine, especially the traditional Chinese medicine (TCM). In contrast to allopathic medicine, TCM seeks to treat the body as a whole, which fosters a cooperative relationship among all elements (molecule, cell, tissue, organ, etc.) involved, leading towards optimal health and wellness. Under the holistic concept, in clinic practice, a large number of effective TCM prescriptions for treating various diseases have been developed since 2,000 years ago, for example, compound Danshen formula [7], Shexiang Baoxin formula [8], Curcumae formula [9], and so forth. Although the concept of holistic medicine has been revealed to some extent in TCM practice, scientific evidence to illustrate the mechanisms of holistic medicine is severely lacking and many key problems associated still exist: for example, (1) in TCM framework, how the body correlates with the external environment and adjusts to it; (2) from molecules to organs, how the body responds to drug treatment and returns to a normal dynamic balance; (3) how the herb system (active ingredients) interacts with the body system, resulting in curing of disease.

In an attempt to uncover the holistic way of TCM from a molecular to systems level, many researchers have done plenty of constructive work over the past years [10, 11]. Representative of typical technologies are chemical [12], pharmacokinetic [9], and pharmacological [13] approaches. These works have, respectively, tended to focus on component identification, drug metabolism, and drug action mechanism investigation when discussing pathological conditions, rather than focusing on the foremost holistic feature of TCM, despite the importance of the latter in physiological phenomena. However, such identified active compounds in TCM may provide clues as to their potential for addressing multiple targets in silico, such as the omic-based ligand-target chemogenomic model (LTC) [14]. Furthermore, mapping of the screened compounds onto their associated biological pathway may suggest potential sites of interaction between TCMderived ingredients and human body. Thus, the integration of the above technologies will provide deep insights into data consolidation and analysis associated with TCM. Once constructed, it will be possible to deconstruct the molecular pathways and open new horizons for disease management, for example, by linking the genes expression levels and their respective targets with the administered TCM active ingredients/metabolites. Such technique will no doubt be favorable for the modernization of TCM and the establishment of the multicomponent, multitargeting strategy as a new paradigm in medicine.
In this study, we develop novel systems pharmacology approach to dissect holistic medicine in treating complex diseases by TCM, which is exemplified by CCDs. Currently, CCDs remain the major cause of death in the United States and constitute $17 \%$ of overall national health expenditures [15] due to the inefficiency of the present world's most advanced molecular targeted therapies in clinical practice. However, the successful applications of TCM in CCDs [7, 9] bring hope to these patients who are trapped into systemic symptoms caused by gene-organ-environment interactions. Interestingly, the pathogenesis of CCDs is consistent with the holistic concept of TCM. To decode the role TCM plays in treating complex diseases, the following steps are proposed: firstly, by applying the TCM active ingredients as baits, we fished and experimentalized the signed drug-target interactions to evaluate the pharmacological actions of drugs at a molecule level; then, at an organ level, we focused on exploring the tissue distribution of targets by a gene extraction method; finally, the translational integrating pathway approach was applied to construct complex diseases therapeutic modules for understanding the mechanism of complex diseases at systems level. As we have seen, this is the first time to illustrate the nature of that holistic medicine which provides effective access to prevention and cure of complex diseases. We believe that holistic medicine of investigating a molecule to systems level therapeutic of disease will facilitate the development of new treatment paradigms for complex diseases in the future.

\section{Materials and Methods}

In this section, we propose a novel systems-based approach which is composed of four modules to explore the nature of holistic medicine (Figure 1). Specifically, we adopt an orally administered Chinese patent medicine Xinnaoxin Pill (Table S1 in Supplementary Material available online at http://dx .doi.org/10.1155/2015/980190) consists of Rhodiola rosea $(R$. rosea), Lycium chinense Miller (L. chinense), and Hippophae rhamnoides Linn. (H. rhamnoides) that has been applied in clinic to treat CCDs, as an example to illustrate the proposed model.

2.1. ADME-Systems Evaluation. An in house in silico ADME (absorption, distribution, metabolism, and elimination) integrative model is developed for evaluating the most important ADME properties of a drug molecule, that is, PreOB (predicts oral bioavailability), PreHIA (predicts human intestinal absorption), PreDL (predicts drug-likeness), and PreBBB (predicts blood-brain barrier) (Figure 2).

2.1.1. PreOB. Owing to the fact that $\mathrm{OB}$ is one of the most important determinants of the dosing regimens for drugs [16], PreOB directs toward identifying final oral compounds in drug prescreening process. PreOB was supported by a dataset of 805 structurally diverse drugs with determination coefficient $\left(R^{2}\right)$ of 0.80 and standard error of estimate (SEE) of 0.31 , which brought P-glycoprotein (P-gp) and cytochrome $\mathrm{P} 450$ s into the construction of the prediction model, which has been successfully applied in many drug screening studies [17]. 


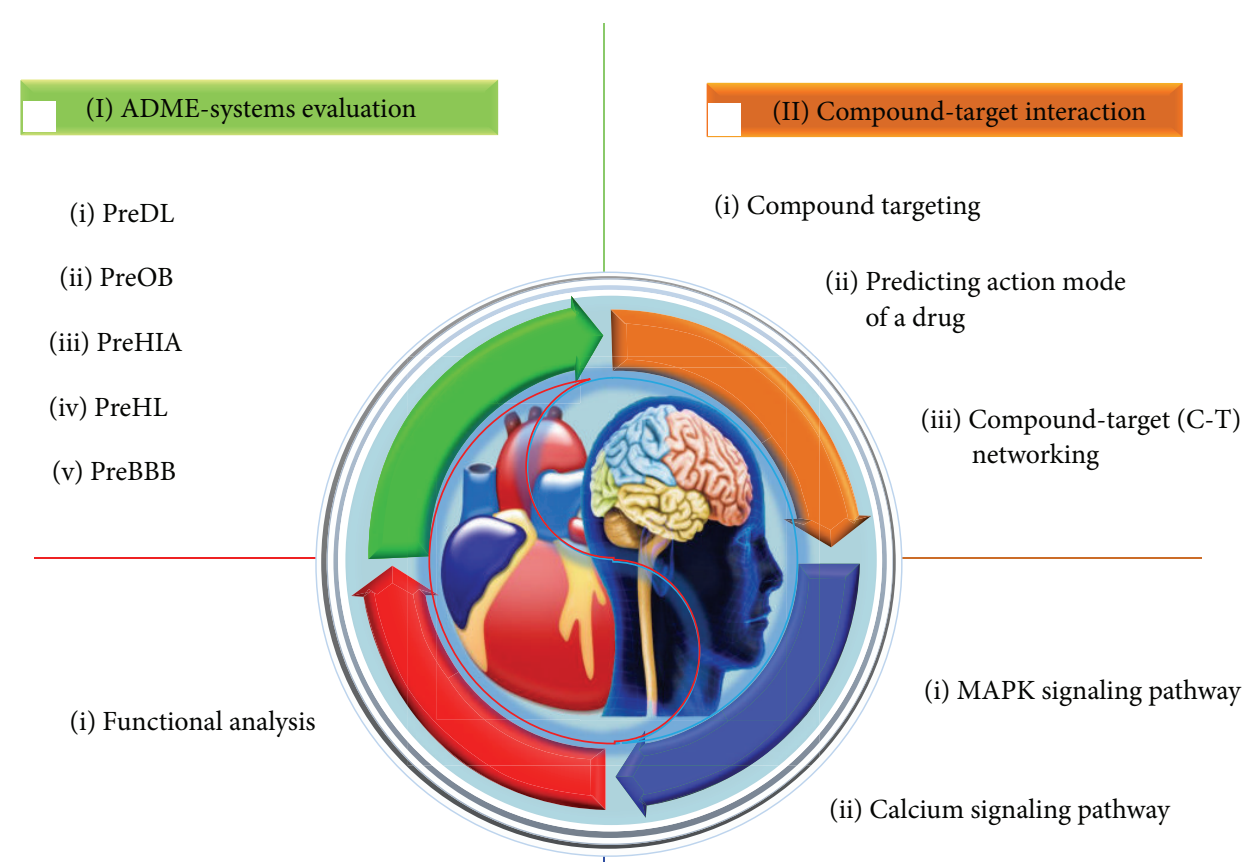

(ii) Target tissue location

(iii) PI3K-Akt signaling pathway

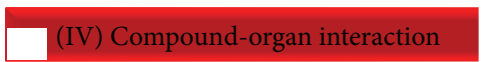

(III) Compound-pathway interaction

FIGURE 1: Workflow for holistic medicine in the treatment of complex diseases.

2.1.2. PreHIA. To predict human intestinal absorption for drugs, a new model is generated by means of the classification-partial least squares (C-PLS) method [18]. PreHIA mainly includes the following three steps.

(1) Data Sets. A total of chemically dissimilar 732 compounds with their quantitative human intestinal absorption percentage values were collected from three studies [19-21]. The compounds are mainly absorbed by passive diffusion and without known issues such as dose-limited, dose-dependent, or formulation-dependent influence. The HIA values of drugs greater than $70 \%$ were deemed as the judging boundary for high absorption and low absorption.

(2) Descriptor Calculation and Selection. To calculate representations of the structural and physicochemical features of the molecules [7], the DRAGON 5.4 program (http://www.talete.mi.it/index.htm) was applied on 929 2D molecular descriptors. Objective feature selection based on forward stepwise algorithm was then employed to discard redundancy and noncontributing descriptors in the descriptors pool and eventually 49 (Table S2) of them were obtained and further applied for C-PLS modeling process.

(3) Model Performance. The dataset is separated into training and test sets by an internal 10 -fold cross-validation. The accuracies for overall, high absorption, and low absorption prediction were used to measure the performance of the PLS model. As a result, the derived model shows impressive performance of prediction for HIA by 5 PLS components, with an overall accuracy of $83.7 \%$, a high absorption prediction accuracy of $81.8 \%$, and a low absorption prediction accuracy of $91.5 \%$ (see details in Figure S1).

2.1.3. PreDL. PreDL was developed to recognize molecules that are "drug-like" and capable of modulating targets, which has been successfully applied in many studies $[9,22]$. The DL values were obtained by calculating the Tanimoto similarity [23] between herbal compounds and the average molecular properties of all compounds in the Drugbank database [24].

2.1.4. PreBBB. To detect whether the biologically active compounds can pass through the blood-brain barrier, an updated model previously constructed by PLS algorithm is developed. PreBBB contains 190 related but chemically diverse compounds which are strong, moderate, or nonpenetrating cross the blood-brain barrier [25].

In this work, the threshold values for the integrative ADME screening system are $\mathrm{OB} \geq 30 \%$, DL $\geq 0.18$, and $\mathrm{HIA} \geq 70 \%$. And the compounds with $\mathrm{BBB}<-0.3$ were considered as nonpenetrating $(\mathrm{BBB}-)$, from -0.3 to +0.3 moderate penetrating $(\mathrm{BBB} \pm)$, and $>0.3$ strong penetrating $(\mathrm{BBB}+)$. Molecules that successfully meet $67 \%(2 / 3)$ of 


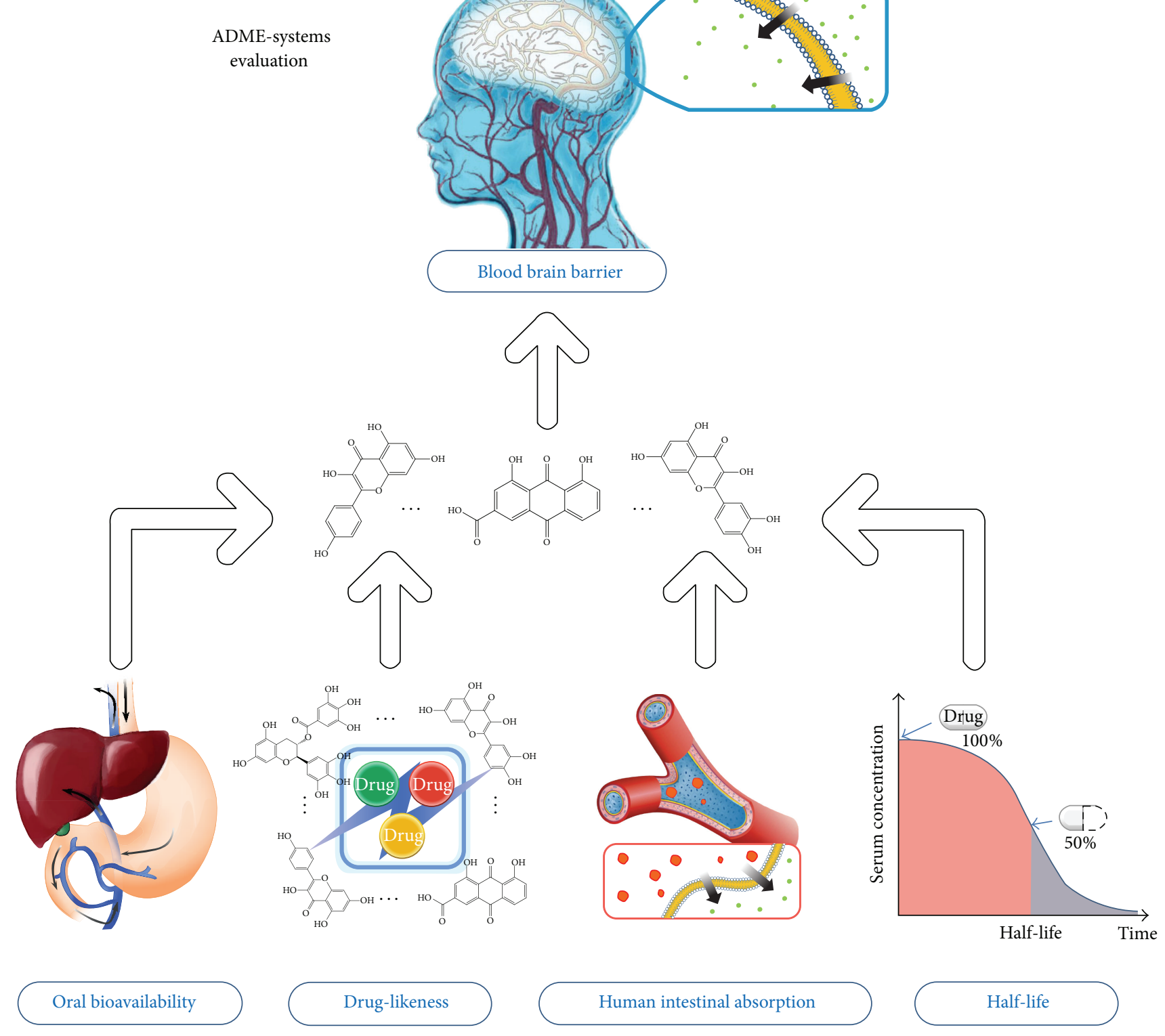

FIgURE 2: ADME-systems evaluation model.

the criteria are nominated as bioactive compounds for further analysis.

2.2. Drug-Target Interaction. For the elucidation of the interactions between target proteins and all drugs, the quantitative analyses of drug targeting, action mode of a drug, drugtarget (D-T) network, and in vitro experimentation were investigated.

2.2.1. Drug Targeting. To obtain cerebrovascular diseases (CBVDs) and CVDs target profiles, a comprehensive drug targeting approach integrating the text mining, database search, and chemometric analysis was applied. First, a fulltext data mining was carried out in TCMSP database (http:// lsp.nwsuaf.edu.cn/tcmsp.php) to derive the molecular target information. Second, a chemical fingerprint-based Similarity Ensemble Approach was applied to obtain the potential targets (http://sea.bkslab.org/search/). Third, an in-house LTC [14] was further introduced for expanding the target pool. The targets from different sources were connected to database UniProt (http://www.uniprot.org/) for target name standardization, which were further subjected to PharmGkb [26], Therapeutic Target Database [27], and the Comparative Toxicogenomics Database [28] to delete noise and errors and to ensure the quality of target database.

2.2.2. PreAM: Predicting Mode of Action for Drugs. In an effort to stringently assess the relationships between compounds and corresponding targets, for the first time, a new PreAM model was built in this work. The data sets of drug 
structures and protein sequences for drug-target interactions (DTIs) with known action modes were retrieved from DrugBank database (http://www.drugbank.ca/, accessed on October 1, 2013). Here, we separated these DTIs into two categories based on their action modes: (1) activated DTIs, where the description label covers "agonist," "activator," "inducer," "stimulator," and "partial agonist"; (2) inhibited DTIs, where the action label contains any of the keywords "inhibitor," "antagonist," "inactivator," "negative modulator," "partial antagonist," "suppressor," and "reducer actions." In total, 6,006 DTIs (including 1,251 activated DTIs and 4,755 inhibited DTIs) were used as benchmark data (Table S3).

To characterize the interactions of drug and protein, drug structures and protein sequences were converted into numerical descriptors by employing DRAGON program (http:// www.talete.mi.it/index.htm) and PROFEAT WEBSEVER (http://jing.cz3.nus.edu.sg/cgi-bin/prof/prof.cgi/), respectively (see details in Table S3). The multiple DTIs were represented by concatenating these chemical and protein descriptors and the minimal-redundancy-maximal-relevance (mRMR) was applied as a variable selection strategy to recognize the best combination descriptors that are most relevant to obtain the models with the highest predictive power. The first 100 descriptors were used in subsequent study.

The random forests (RF) algorithm (http://www.stat .berkeley.edu/users/breiman/) was trained to generate a nonlinear classifier tailored to DTIs with known action modes. The accuracies to overall, activation, inhibition were used to measure the performance of the model. The derived model shows impressive performance of prediction for drug-target interactions, with an overall accuracy of $97.3 \%$, an activated prediction accuracy of $87.7 \%$, an inhibited prediction accuracy of $99.8 \%$. (see details in Figure S2).

2.2.3. Drug-Target (D-T) Network. To characterize the multicomponent therapeutic features in the treatment of CBVDs and CVDs, a drug-target network is generated, in which a compound and a target are linked if a compound targets a known protein. The compounds are the ones which are screened out from the ADME systems, with their targets derived from the above targeting process. The bipartite graphs are visualized and analyzed by Cytoscape version 2.8.1 [29].

2.2.4. Experimental Validation. To validate the practicability and efficiency of above methods, the inhibitory effects of drugs on predicted targets were quantified by ligand-binding assays according to the manufacturer's instructions. The drug-target interactions were selected at random and readily available on the market, respectively. Compounds rhodiosin, astragalin, rutin, quercetin, astragalin, and kaempferol-3glucoside were from Yitai Technology Ltd. (Wuhan, China). Targets PIM1 and PTGS1 were respectively purchased from Cayman Chemical, Ann Arbor, MI, USA and CycLex, Japan. The purity of all the compounds is $>98 \%$. All drugs were dissolved in DMS and freshly prepared due to loss of activity under long-term storage. $\mathrm{IC}_{50}$ values were determined using the Bliss method with three independent determinations.
2.3. Drug-Pathway Interaction. To investigate the biological effects exerted on the pathway level, an incorporated "CCDs pathway" was assembled based on the current knowledge of CCDs pathology. First, the obtained target profiles were organized into several pathways by mapping them onto KEGG database (http://www.genome.jp/kegg/). Then, the pathways, which are not directly related to CCDs, were removed according to the pathological and clinical data. Finally, a relatively complete CCDs pathway was manually synthesized, including MAPK signaling pathway, calcium signaling pathway, and PI3K-Akt signaling pathway. By adopting the $\varphi_{p p^{\prime}}$ expression in HmSP [30], we further make a nearness analysis on the correlativity between the obtained targets and the "CCDs pathway" related proteins in the protein-protein interaction (PPI) network.

2.4. Drug-Organ Interaction. To understand the underlying connections of CBVDs and CVDs on organ level, it is essential to check the functional and tissue expression profile of the protein targets of the two related diseases. Firstly, we enrich the overrepresented gene ontology (GO) terms and check the expressed tissue distribution in the obtained target networks [31]. For GO analysis, the biological process of GO vocabulary (GOBP) was highlighted. The target tissue distribution was determined based on the microarray analyses data of different tissue types lodged in the BioGPS bank (accessible at http://biogps.org/), an extensible and customizable portal for querying and organizing gene annotation resources. The expressions for targets tissue distribution are as follows:

$$
\Omega=\left\{t_{1}, t_{2}, \ldots, t_{n}\right\}
$$

where $t$ is the human body organization and $\Omega$ is the set of tissues:

$$
H_{i}=\left(h_{i t_{1}}, h_{i t_{2}}, \ldots, h_{i t_{n}}\right)
$$

where $h$ is the tissue-specific pattern of mRNA expression of a target and $H_{i}$ is the mRNA expression set of a target in $\Omega$ :

$$
\overline{h_{i}}=\frac{\sum_{i=1}^{n} h_{i}}{n},
$$

where $\overline{h_{i}}$ is the average expression quantity of a target in tissues and $n$ is the number of tissues:

$$
A_{i}=\left\{t \in \Omega \mid t>\overline{h_{i}}\right\},
$$

where $A_{i}$ is the tissue localization of a target.

\section{Results}

All the compounds, biologically active compounds, and targets for Xinnaoxin Pill have been uploaded to our TCMSP database. All readers can freely access the TCMSP database.

3.1. Multicomponent Therapeutics. As shown in Table 1, 18 compounds in Xinnaoxin Pill match all the filter criteria in Section 2.1. There are 1, 5, and 9 compounds that exist only in $R$. rosea, L. chinense, and $H$. rhamnoides, respectively. 


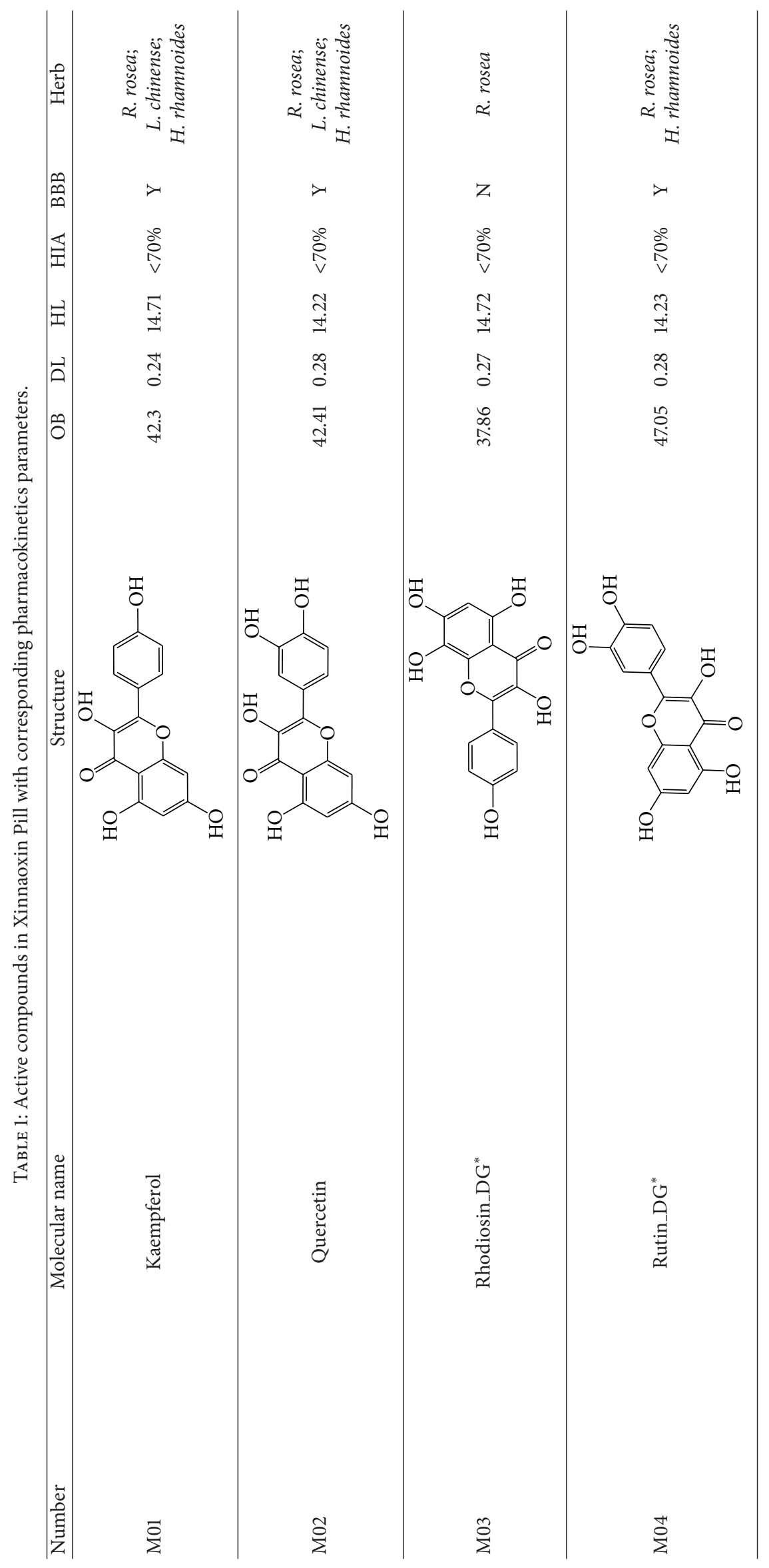




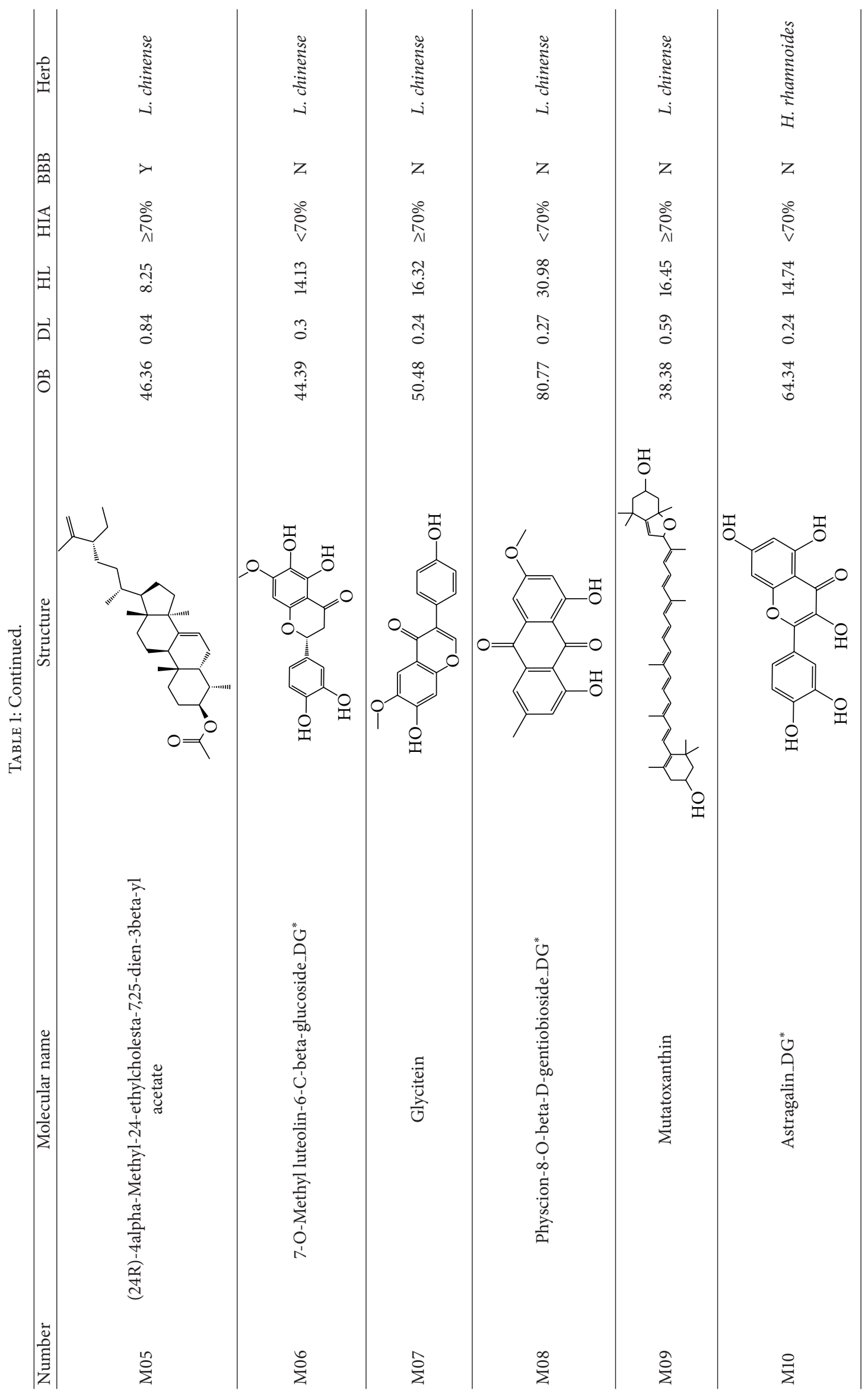




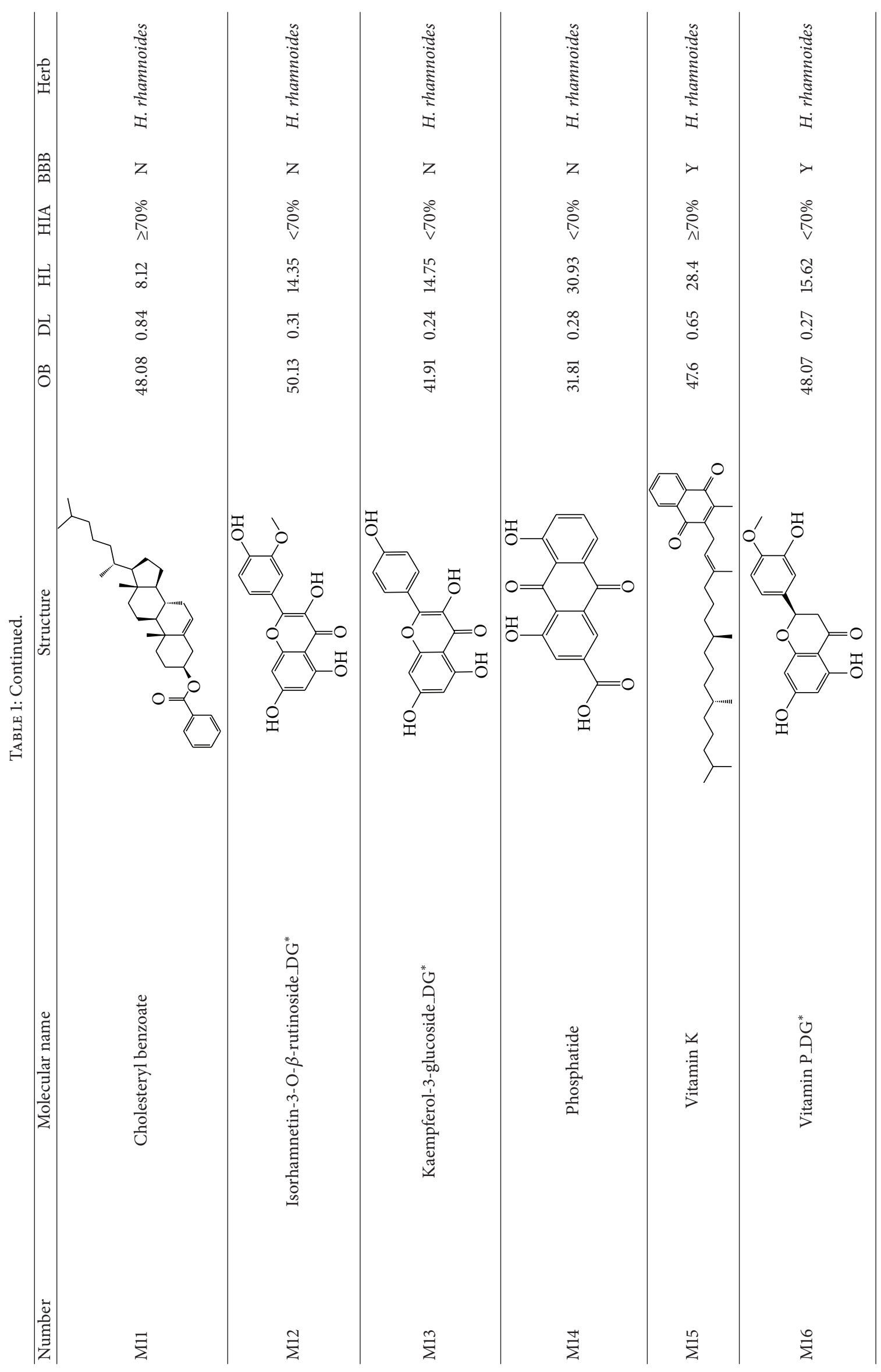




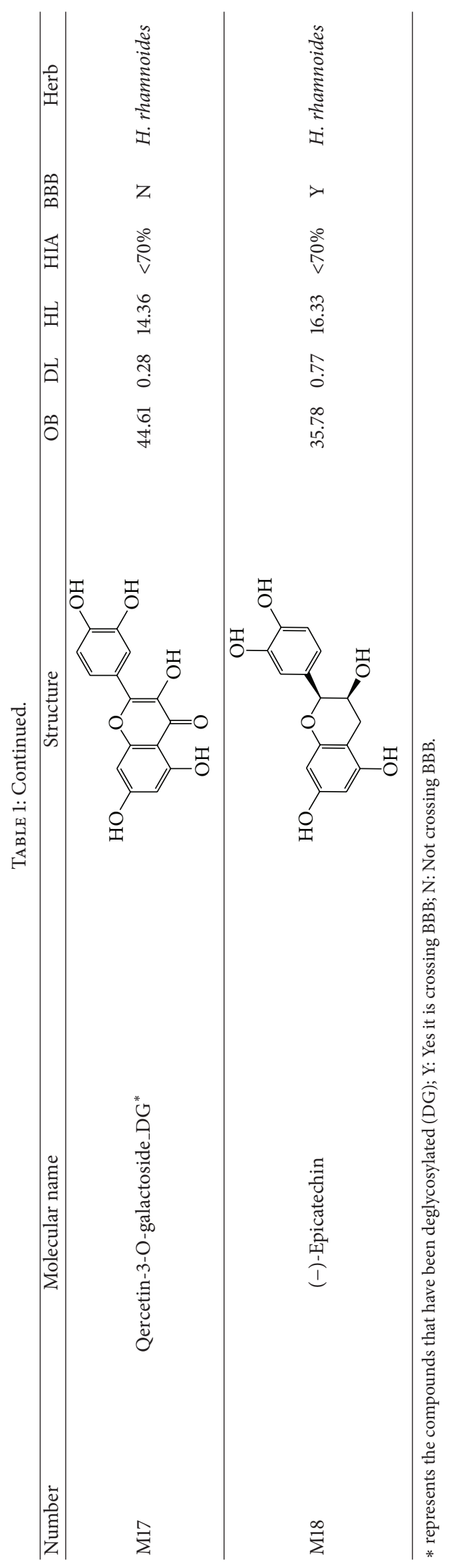




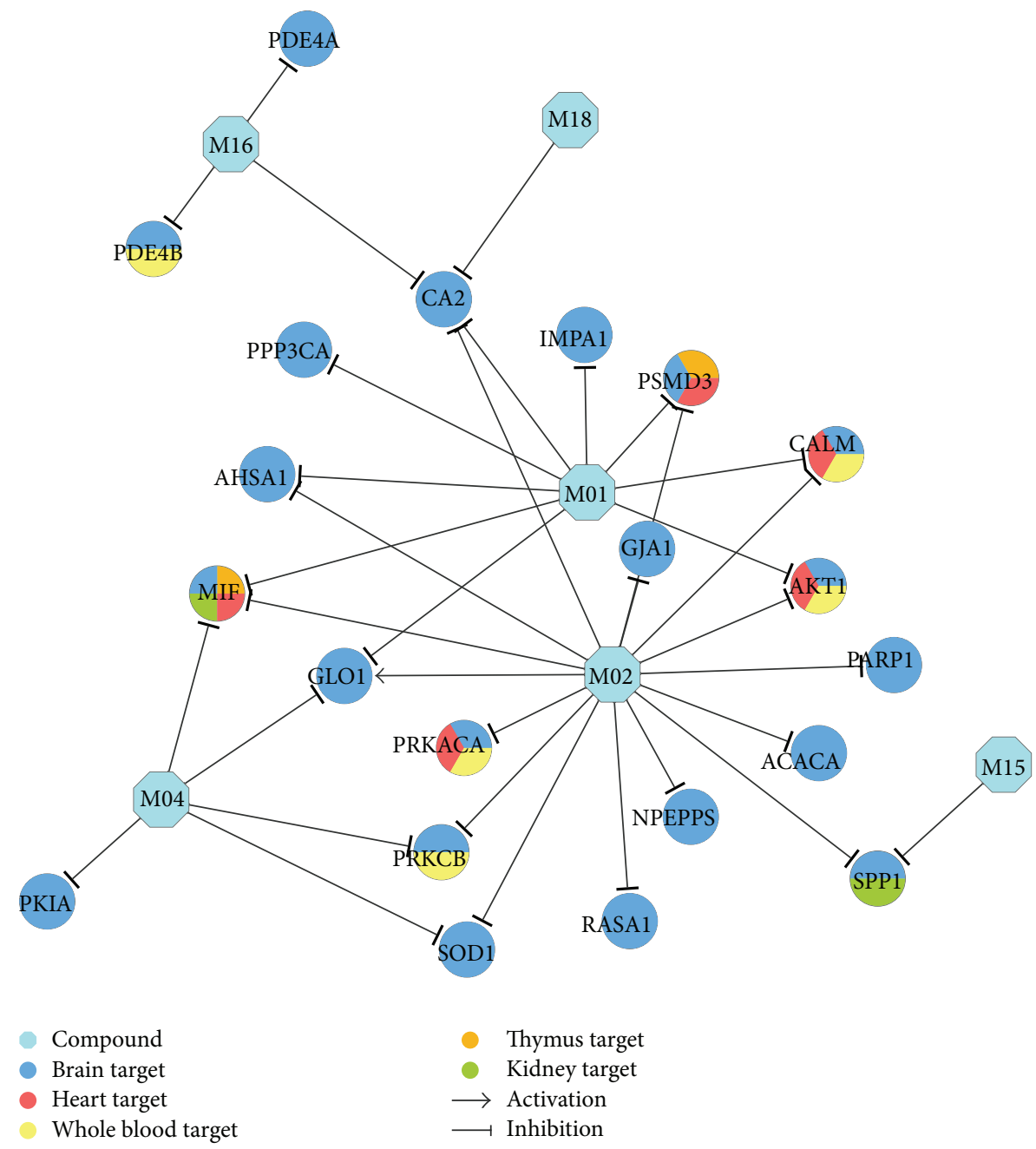

Figure 3: CBVDs therapeutic compound-target network. A compound node and a protein node are linked if the protein is targeted by the corresponding compound. Node size is proportional to its degree. The letters are target labels. Arrows indicate activation and T-arrows represent inhibition.

3.1.1. $R$. rosea. The bioactive ingredients in $R$. rosea are kaempferol, quercetin dehydrate, rhodiosin, and rutin, which possess both therapeutic effects for CBVDs and CVDs. For kaempferol, it was reported that persons with high kaempferol intakes normally have low incidence of CBVDs [32]. Quercetin can prevent heart disease [33] and rhodiosin shows a strong antioxidant activity [34] and thus remedies coronary artery disease [35]. In addition, Harvard researchers recently found that rutin has potent anticlotting powers for preventing heart attack [10].

3.1.2. L chinense. The main active substances in this herb are glycitein, mutatoxanthin, quercetin, and so forth (Table 1). Glycitein is suggested to be a potential alternative to estrogen therapy in the treatment of CVDs [36]. Mutatoxanthin may be scavenger of reactive species, thus showing antioxidant activity [37], which is similar for quercetin cardioprotective activities supported by in vitro and animal studies [38, 39].
3.1.3. $H$. rhamnoides. The potential active substances are astragalin, flavoxanthin, vitamin $\mathrm{K}$, and so on (Table 1). Astragalin has positive myocardial inotropic effects by inhibiting the platelet aggregation [40]. Flavoxanthin shows preferable activity in reduction of degenerative heart disease [41]. Vitamin K plays a preventive role in coronary artery calcification for the properties of matrix Gla protein.

Of the 18 bioactive compounds in Xinnaoxin Pill, eight compounds can freely cross the $\mathrm{BBB}$ (Table 1) and act as the main therapeutics for the treatment of CBVDs. The other BBB nonpermeable molecules are considered to combat CVDs.

3.2. Drug-Target Interaction. 595 D-T interactions (Table S4) data between 18 compounds and 218 targets were generated by the method in Section 2.2. In all the interactions, 35 interactions modulate the 24 (Table 2) brain high-abundant targets through 6 compounds that can freely cross BBB. The 35 interactions were shown in Figure 3, which might explain 
TABLE 2: The CBVDs target information.

\begin{tabular}{|c|c|c|}
\hline Gene name & Protein name & Degree \\
\hline CA2 & Carbonic anhydrase 2 & 4 \\
\hline GLO1 & Lactoylglutathione lyase & 3 \\
\hline MIF & $\begin{array}{l}\text { Macrophage migration inhibitory } \\
\text { factor }\end{array}$ & 3 \\
\hline AHSA1 & $\begin{array}{l}\text { Activator of } 90 \mathrm{kDa} \text { heat shock protein } \\
\text { ATPase homolog } 1\end{array}$ & 2 \\
\hline AKT1 & $\begin{array}{l}\text { RAC-alpha serine/threonine-protein } \\
\text { kinase }\end{array}$ & 2 \\
\hline CALM & Calmodulin & 2 \\
\hline PRKCB & Protein kinase $\mathrm{C}$ beta type & 2 \\
\hline PSMD3 & $\begin{array}{l}26 \mathrm{~S} \text { proteasome non-ATPase } \\
\text { regulatory subunit } 3\end{array}$ & 2 \\
\hline SOD1 & Superoxide dismutase [Cu-Zn] & 2 \\
\hline SPP1 & Osteopontin & 2 \\
\hline ACACA & Acetyl-CoA carboxylase 1 & 1 \\
\hline GJA1 & Gap junction alpha-1 protein & 1 \\
\hline IMPA1 & Inositol monophosphatase & 1 \\
\hline NPEPPS & Puromycin-sensitive aminopeptidase & 1 \\
\hline PARP1 & Poly [ADP-ribose] polymerase 1 & 1 \\
\hline PDE4A & $\begin{array}{l}\text { cAMP-specific } 3^{\prime}, 5^{\prime} \text {-cyclic } \\
\text { phosphodiesterase } 4 \mathrm{~A}\end{array}$ & 1 \\
\hline PDE4B & $\begin{array}{l}\text { cAMP-specific } 3^{\prime}, 5^{\prime} \text {-cyclic } \\
\text { phosphodiesterase } 4 \mathrm{~B}\end{array}$ & 1 \\
\hline PKIA & $\begin{array}{l}\text { cAMP-dependent protein kinase } \\
\text { inhibitor alpha }\end{array}$ & 1 \\
\hline РPР3СA & $\begin{array}{l}\text { Serine/threonine-protein phosphatase } \\
\text { 2B catalytic subunit alpha isoform }\end{array}$ & 1 \\
\hline PRKACA & $\begin{array}{l}\text { cAMP-dependent protein kinase } \\
\text { catalytic subunit alpha }\end{array}$ & 1 \\
\hline RASA1 & Ras GTPase-activating protein 1 & 1 \\
\hline
\end{tabular}

why Xinnaoxin Pill can be used for the treatment of CBVDs. Other interactions were described in Figure 4 and deemed to decode pharmacological effects for CVDs.

With respect to action modes of the 595 DTIs, 546 are inhibited DTIs, and the others are activated DTIs. Among these DTIs, many of them are identical with those reported in the literature, such as TNF [42] and ESR1 [43]. These results indicate the reliability of the PreAM model.

3.2.1. D-T $T_{1}$ Network for Curing CBVDs. Figure 3 shows that there are 24 targets of CBVDs in the $\mathrm{C}_{-} \mathrm{T}_{1}$ network modulated by 6 compounds that can freely cross the BBB. Among the targets, CA2 (carbonic anhydrase 2) displays the highest degree ( $\mathrm{DD}=9$ ), followed by MIF (macrophage migration inhibitory factor, DD = 5), GLO1 (lactoylglutathione lyase, DD = 4), IMPA1 (inositol monophosphatase $1, \mathrm{DD}=4$ ), PRKACA (camp-dependent protein kinase catalytic subunit alpha, DD = 3), and so forth. All these results indicate that the three herbs $R$. rosea, $L$. chinense, and $H$. rhamnoides in Xinnaoxin Pill can interact with the cerebrovascular diseaseassociated proteins. For instance, (1) CA2 plays a critical role in the cerebrovascular disease that triggered Alzheimer's disease [44]. Thus, the inhibition of CA2 by M01 reduces the chances of Alzheimer's disease after cerebrovascular disease; (2) studies suggest that MIF is upregulated in the brain after cerebral ischemia, and disruption of the Mif gene in mice leads to a smaller infarct volume and better sensory-motor function after transient middle cerebral artery occlusion [45]. Similarly, M01 is predicted to inhibit MIF, also responsible for CBVDs; (3) it has been reported that pharmacological inhibition of GLO1 could exacerbate thermal hyperalgesia [46]. Here, we find that molecule M02 (in all three herbs) is able to activate GLO1, thus exerting clinical effect on CBVDs.

Interestingly, the topology of the network does not have a clear bias toward PRKCB (protein kinase $\mathrm{C}$ beta type, $\mathrm{DD}=$ 2), SPP1 (osteopontin, DD = 2), CALM (calmodulin, DD = 2), and so forth. They are all attractive therapeutic targets curing CBVDs. For example, it has been reported that inhibition of PRKCB increased $\mathrm{BBB}$ permeability during hyperglycemic stroke and prevents edema formation in vivo [47]. And this target was predicted to be inhibited by M02 in herbs of $R$. rosea, L. chinense, and $H$. rhamnoides, further highlighting the efficiency of Xinnaoxin Pill in treatment of CBVDs. SPP1 is a multifunctional protein which has shown neuroprotective properties in animal models of cerebral ischemia [48]. Nevertheless, its role in acute human stroke has not been recognized. Here we found that M15 (vitamin $\mathrm{K}$ in $H$. rhamnoides) is capable of inhibiting SPP1, thus elucidating the role of SPP1 in ischemic stroke pathophysiology.

3.2.2. $D-T_{2}$ Network for Combating CVDs. Figure 4 provides a network-level understanding of the mechanism of Xinnaoxin Pill for treating CVDs. It shows that targets (Table 3) with high degree of connectivity ( $>3)$, such as ESR1 (estrogen receptor- $\alpha, \mathrm{DD}=16$ ), ESR2 (estrogen receptor$\beta, \mathrm{DD}=15$ ), AR (androgen receptor, $\mathrm{DD}=14$ ), and PIM1 (serine/threonine-protein kinase pim-1, DD $=15$ ), are all therapeutic targets of CVDs. For example, (1) data from animal model and clinical studies support the associations between polymorphisms in both ESRs and CVDs $[49,50]$. M02 (quercetin) is shown to block ESRs and AR indicating the effectiveness of the treatment. But unlike M02, M03 (rhodiosin in R. rosea) has active effects on the ESRs. (2) In humans, PIM1 is normally expressed in the cytoplasm of cardiomyocytes in adult myocardial tissue. In fact, study has led to the discovery that PIM1 promotes cardioprotective signaling and enhances cardiac structure and function after pathological injury [51]. In this paper, we found that M03 can inhibit PIM1, which is worth more attention for future studies.

3.2.3. Ligand-Target Analysis. As shown in Table 4, the experimental $\mathrm{IC}_{50}$ values from the inhibition test yield an estimate for antigen-antibody reactions, which agree well with those derived from the PreAM model. Compounds of rhodiosin, 


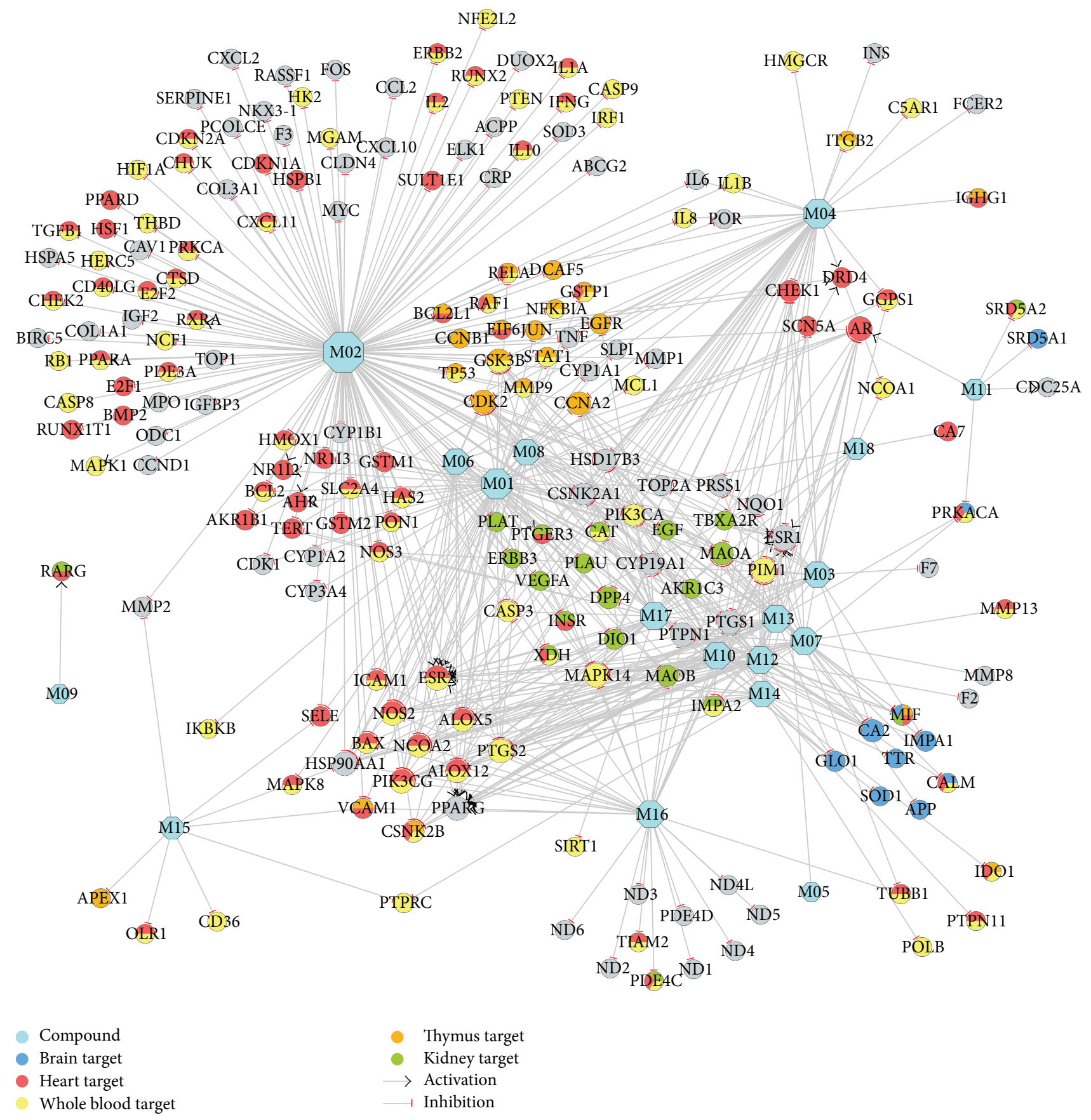

FIGURE 4: CVDs therapeutic compound-target network. A compound node and a protein node are linked if the protein is targeted by the corresponding compound. Node size is proportional to its degree. The letters are target labels. Arrows indicate activation and T-arrows represent inhibition.

astragalin, and rutin were tested in PIM1 inhibition assays and proved to be active as inhibitors of it. Although the data highlighted that astragalin $\left(\mathrm{IC}_{50}=62 \mu \mathrm{M}\right)$ and rutin $\left(\mathrm{IC}_{50}=57 \mu \mathrm{M}\right)$ turned out to be more active than rhodiosin $\left(\mathrm{IC}_{50}=96 \mu \mathrm{M}\right)$, indicating that rhodiosin can also repress the vitality of PIM1 as well as astragalin, and rutin. The highly effective compound quercetin bound to PTGS1 with an $\mathrm{IC}_{50}$ of $82 \mu \mathrm{M}$ in a manner characteristic of antagonists. To further define its mode of action, we tested the $\mathrm{IC}_{50}$ of compounds of astragalin and kaempferol-3-glucoside. Astragalin with an apparent $\mathrm{IC}_{50}$ of about $43 \mu \mathrm{M}$ had obvious inhibitory effect on the target PTGS1. Interestingly, kaempferol-3-glucoside $\left(\mathrm{IC}_{50}=74 \mu \mathrm{M}\right)$ also showed the suppression effect of PTGS1. These findings indicated that the PreAM model can be used accurately and conveniently in assessing the action mode of drug-target interactions. 
TABLE 3: The CVDs target information.

\begin{tabular}{|c|c|c|}
\hline Gene name & Protein name & Degree \\
\hline ESR1 & Estrogen receptor & 15 \\
\hline ESR2 & Estrogen receptor beta & 14 \\
\hline PIM1 & $\begin{array}{l}\text { Proto-oncogene serine/threonine-protein } \\
\text { kinase Pim-1 }\end{array}$ & 14 \\
\hline $\mathrm{AR}$ & Androgen receptor & 13 \\
\hline HSP90AA1 & Heat shock protein HSP 90-alpha & 12 \\
\hline MAPK14 & Mitogen-activated protein kinase 14 & 12 \\
\hline NOS2 & Nitric oxide synthase, inducible & 12 \\
\hline PIK3CG & $\begin{array}{l}\text { Phosphatidylinositol-4,5-bisphosphate } \\
\text { 3-kinase catalytic subunit gamma isoform }\end{array}$ & 12 \\
\hline PPARG & $\begin{array}{l}\text { Peroxisome proliferator-activated } \\
\text { receptor gamma }\end{array}$ & 12 \\
\hline PTGS1 & Prostaglandin G/H synthase 1 & 12 \\
\hline PTGS2 & Prostaglandin G/H synthase 2 & 12 \\
\hline PTPN1 & $\begin{array}{l}\text { Tyrosine-protein phosphatase } \\
\text { non-receptor type } 1\end{array}$ & 12 \\
\hline $\mathrm{CDK} 2$ & Cell division protein kinase 2 & 11 \\
\hline GSK3B & Glycogen synthase kinase- 3 beta & 11 \\
\hline ALOX12 & Arachidonate 12-lipoxygenase, 12S-type & 10 \\
\hline CCNA2 & Cyclin-A2 & 10 \\
\hline ALOX5 & Arachidonate 5-lipoxygenase & 9 \\
\hline CA2 & Carbonic anhydrase 2 & 9 \\
\hline MAOA & Amine oxidase [flavin-containing] A & 9 \\
\hline NCOA2 & Nuclear receptor coactivator 2 & 9 \\
\hline CSNK2A1 & Casein kinase II subunit alpha & 7 \\
\hline CSNK2B & Casein kinase II subunit beta & 7 \\
\hline CYP19A1 & Cytochrome P450 19A1 & 7 \\
\hline $\mathrm{DPP} 4$ & Dipeptidyl peptidase 4 & 7 \\
\hline HSD17B3 & Estradiol 17-beta-dehydrogenase 3 & 7 \\
\hline PIK3CA & $\begin{array}{l}\text { Phosphatidylinositol-4,5-bisphosphate } \\
\text { 3-kinase catalytic subunit alpha isoform }\end{array}$ & 7 \\
\hline CHEK1 & Serine/threonine-protein kinase Chk1 & 6 \\
\hline MAOB & Amine oxidase [flavin-containing] B & 6 \\
\hline CASP3 & Caspase-3 & 5 \\
\hline IMPA2 & Inositol monophosphatase 2 & 5 \\
\hline MIF & Macrophage migration inhibitory factor & 5 \\
\hline PRSS1 & Trypsin-1 & 5 \\
\hline BAX & Apoptosis regulator BAX & 4 \\
\hline GLO1 & Lactoylglutathione lyase & 4 \\
\hline IMPA1 & Inositol monophosphatase & 4 \\
\hline TOP2A & DNA topoisomerase 2-alpha & 4 \\
\hline VCAM1 & Vascular cell adhesion protein 1 & 4 \\
\hline AKR1B1 & Aldose reductase & 3 \\
\hline CAT & Catalase & 3 \\
\hline
\end{tabular}

TABLE 4: $\mathrm{IC}_{50}$ values for the randomly selected drug-target interactions.

\begin{tabular}{lccc}
\hline Number & $\begin{array}{c}\text { Target gene } \\
\text { name }\end{array}$ & Drug name & $\begin{array}{c}\mathrm{IC}_{50}(\mu \mathrm{M}): \\
\text { mean } \pm \text { SD }\end{array}$ \\
\hline 1 & PIM1 & Rhodiosin & $96 \pm 3.2$ \\
2 & PIM1 & Astragalin & $62 \pm 9.7$ \\
3 & PIM1 & Rutin & $57 \pm 5.8$ \\
4 & PTGS1 & Quercetin & $464 \pm 6.4$ \\
5 & PTGS1 & Astragalin & $143 \pm 7.0$ \\
6 & PTGS1 & Kaempferol-3- & $181 \pm 11.5$ \\
\hline
\end{tabular}

3.3. Multipathways Regulations for Disease Treatment. An incorporated "CCDs pathway" was assembled based on the current knowledge of CCDs pathology. Of the 218 target proteins, 210 can be mapped onto the pathway. They display extremely significantly close functional linkage correlation to the CCDs pathway related proteins (ultimate nearness = 0.034 , nearness $=0.008, P \ll 0.01$ ). As shown in Figure 5, the CCDs pathway can be divided into several therapeutic modules, such as inflammation, contraction, PGI2 production, proliferation, DNA repair, sustained angiogenesis, and evading apoptosis. As an illustration, three representative therapeutic modules were described in detail to clarify its therapeutic mechanism.

3.3.1. Inflammation Module. Some targets marked in the MAPK signaling pathway are involved in inflammation process, indicating that the anti-inflammatory action is important for the treatment of CCDs. As shown in Figure 5, M02 can activate and inhibit protein TGFB1 and TNF, respectively. In fact, TGFB1 is a multifunctional cytokine involved in the regulation and proliferation of cells. Cytokine promotes differentiation of leucocytes but has inhibitory effects on proliferation of $\mathrm{T}$ lymphocytes and activation of macrophages, suggesting a regulatory role in inflammatory states [52]. TNF promotes inflammation by stimulation of capillary endothelial cell proinflammatory responses and thereby provides leukocyte adhesion and infiltration into the ischemic brain. All this suggests that agents that suppress TNF's production or actions will reduce leukocyte infiltration into ischemic brain regions and thereby diminish the extent of tissue loss [53].

3.3.2. Cardiac Contractility-Associated Module. Calcium signaling pathway might be perturbed by herbal ingredients and produce contraction function, as displayed in Figure 5. For example, ligand M02 is shown with a reduction of PRKCA protein levels and an augmentation of ERBB2, respectively. PRKCA is a fundamental regulator of cardiac contractility and $\mathrm{Ca}^{2+}$ handling in myocytes. Research suggests that hypercontractility caused by Prkca deletion protects against heart failure induced by pressure overload [54]. ERBB2 is essential in the prevention of dilated cardiomyopathy, which is required for maintenance of cardiac contractility [55]. In 


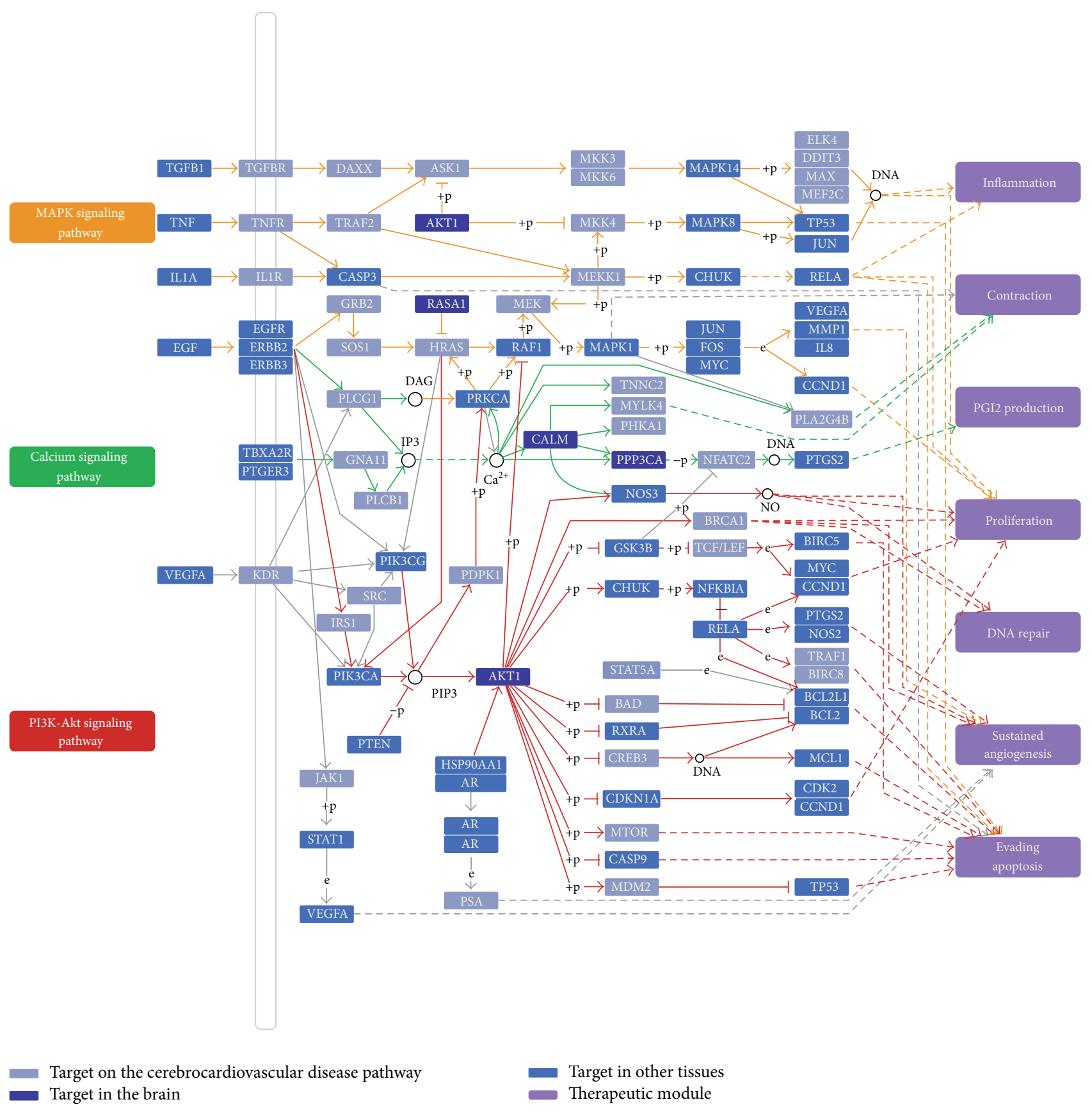

FIGURE 5: Cerebro-CVD pathway and therapeutic modules.

addition, M12 serves as an activator of CALM. It is shown that CALM is intrinsically dependent on changes in intracellular $\mathrm{Ca}^{2+}$ concentration, which regulates cardiac contractility [56]. All these indicate that Xinnaoxin Pill treats the CCDs through one way of promoting cardiac contractility.

3.3.3. Sustained Angiogenesis-Associated Module. Inadequate blood supply to the heart resulting from insufficient angiogenesis is a hallmark feature of many CVDs. In this situation, promoting angiogenesis is an effective therapeutic program both in the repair process of damaged tissue and in the formation of collateral response to tissue ischemia. As marked in Figure 5, some targets involved in sustained angiogenesis are modulated by the herbal drug through acting on PI3K-Akt signaling pathway. M08 and M02 were observed to increase PTGS2 and NOS3 level, respectively. In addition, M10 was also shown with an increment of NOS2. Recent evidence demonstrates that the inhibition of PTGS2 leads to restricted angiogenesis and downregulates production of proangiogenic factors [57]. NOS3 is a mediator of angiogenesis due to the production of $\mathrm{NO}$, which has been shown to modulate angiogenesis in vitro and in vivo [58]. 


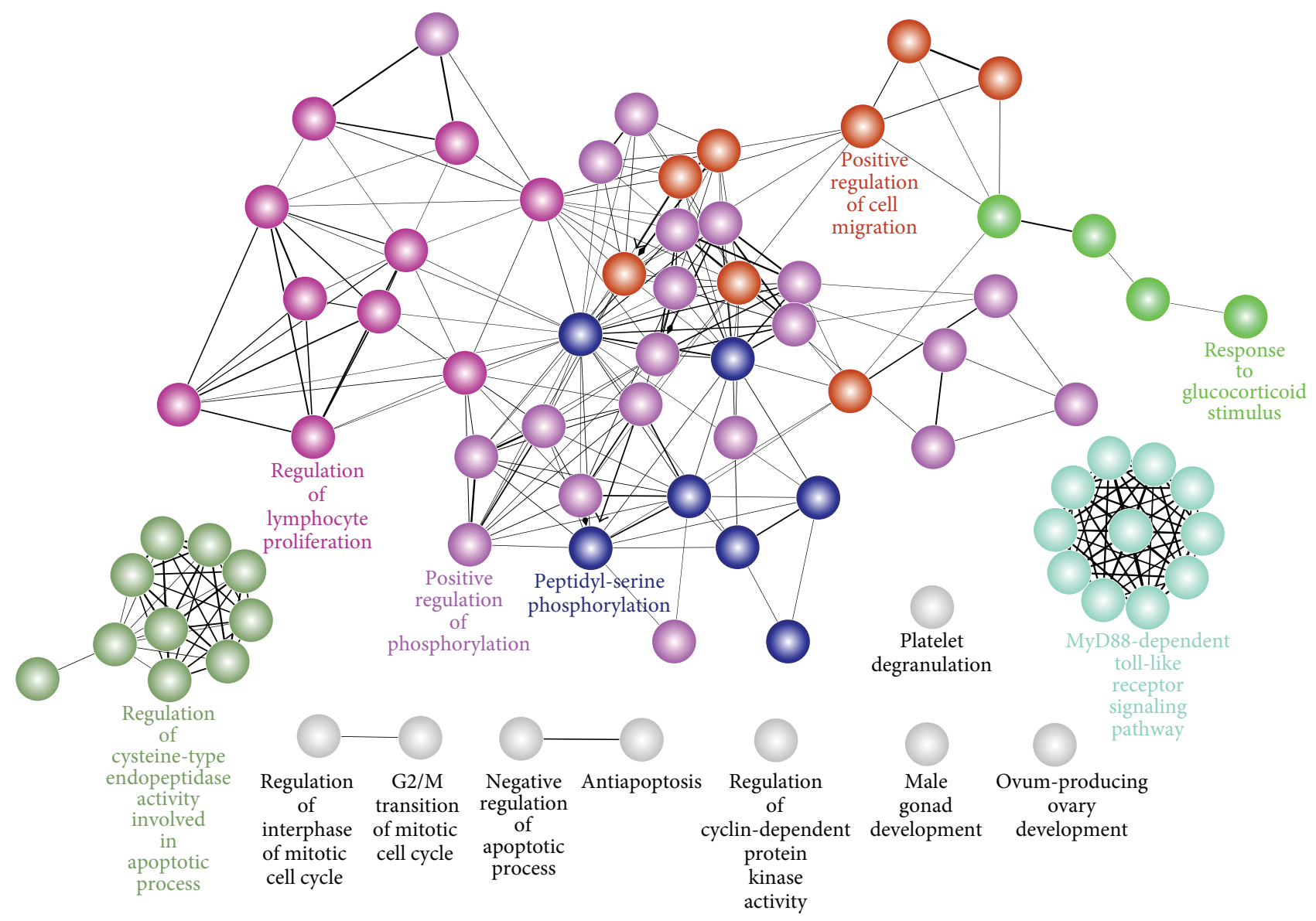

FIGURE 6: Functional grouped network for the targets of Xinnaoxin Pill. Functionally related groups partially overlap, only the label of the most significant term per group is displayed.

Another angiogenesis modulator is NOS2, which appears to exert a direct effect on several angiogenic factors such as VEGF [59]. As stated, angiogenesis is a complex process where several proteins converge and the angiogenesis-based treatment protocol for CCDs that target these proteins will have modest yields and satisfactory results.

\subsection{Multiple-Target/Organ Cooperation for Disease Treatment}

3.4.1. Function Cooperation. GOBP analysis shows that these targets were enriched to regulate the following biological processes: positive regulation of phosphorylation, MyD88dependent toll-like receptor signaling pathway, platelet degranulation, positive regulation of cell migration, peptidylserine phosphorylation, and so forth (Figure 6). These processes take on different functionality and the mutual cooperation with each other yields an effective therapy for complex diseases.

For example, (1) phosphorylation was the most important protein posttranslational modification which is involved in the regulation of diverse biological processes [60], such as endothelial dysfunction [61]. Dysfunction of NOS3 is implicated as a contributing factor in these varied disease states [62]. (2) Toll-like receptors (TLRs) are differently expressed by vessels within normal human vasculature.
Atherosclerosis development in murine models has largely been shown to be associated with increased expression of TLR-2 and TLR-4 [63]. (3) Platelets play a pivotal role in thromboembolism, and a better understanding of the cellular mechanisms involved in platelet activation in ischaemic cerebrovascular disease should improve our understanding of the pathogenesis of stroke and provide opportunities for improved secondary prevention [64]. (4) Cell motility is an important part of many physiological processes such as angiogenesis. Endothelial cell migration and proliferation are central to the process of new blood vessel formation [65].

3.4.2. Target Tissue Location. Microarray analyses of mRNA expression showed that, among the 218 targets, 211 of them had their expression profiles in 84 normal tissues (Table S5). According to BioGPS database, the 211 targets are ubiquitous in human tissues at differing levels. And importantly, we compared expression patterns across different tissues and observed that 24 targets in Table 2 contain higher mRNA expression in brain than the average value of 84 tissues for each target. The 24 brain high-abundant targets are considered as therapeutic target for CBVDs and other lowabundant or hardly expressed 194 targets are thought to be associated with CVDs. 


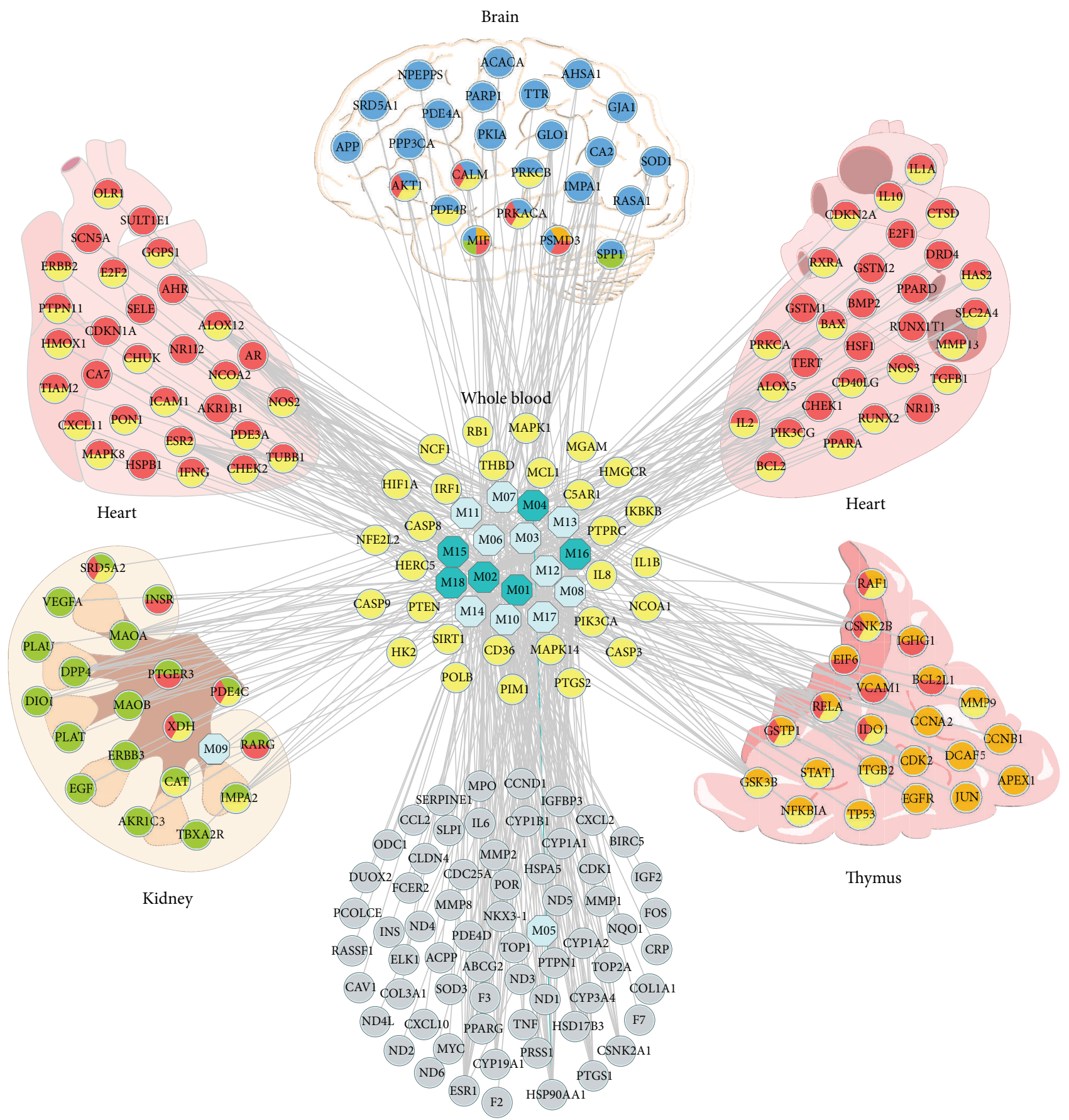

Other tissue
- $\mathrm{BBB}$ impenetrable molecules
Whole blood target
- BBB penetrable molecule
- Thymus target
- Brain target
- Kidney target
Heart target
Other tissue target

FIgURE 7: Target organ location map. The node pie chart represents the organs in which each target is located.

Figure 7 shows the tissue distribution network of the 211 targets based on their expression patterns. The networks are divided into a few tissue modules, including brain, heart, kidney, thymus, and whole blood. Meanwhile, the targets in whole blood are linked with tissues in any form. Our results imply that these tissues are closely related to CCDs and the whole blood guides as a bridge of these tissues. For example, many of the strokes involved areas of the brain 
involved in motor control, such as the basal ganglia [66]. Heart rate has been shown to be an important predictor of mortality in CVDs [67]. Cardiovascular morbidity and mortality in patients with chronic kidney disease (CKD) are high, and the presence of CKD worsens outcomes of CVDs [68]. Patients with thymus hypoplasia have a very high incidence of congenital heart disease [69].

\section{Discussion and Conclusion}

Medicine is undergoing a revolution that will transform the practice of healthcare in virtually every way. This revolution is emerging from the convergence of systems pharmacologya systems approach to medicine. Holistic medicine is the art and science of healing that addresses care of the whole body, especially complex diseases. It promises to (1) define the components of the system, (2) clarify how these components interact with one another, (3) stratify complex diseases into their distinct subtypes for an impedance match against proper drugs, and (4) provide deep insights into disease mechanisms [70].

The workflow for TCM-based holistic medicine constructs an easily-interpretable map that could facilitate understanding how the holistic medicine through systems pharmacology means to remedy chronic conditions from a molecular to systems level, which provides guidance for ascertaining complicated nosogenesis, systems cure strategies, and new drug development.

Generally, the treatment of holistic medicine is chartered as multicomponent therapeutics, multitarget/pathways regulations, and multiorgan cooperation. To define the components of the system, we developed a novel systems pharmacology approach integrating with multiple techniques including ADME-systems evaluation, drug targeting, and target tissue distributing. With the aid of ADME-systems evaluation, 18 active compounds were detected. And these compounds could interact with 218 locations various targets (several times of the compounds) by drug targeting, indicating the multicomponent therapeutics and multitarget regulations characteristic.

To clarify how these components interact with one another, a PreAM model was built to evaluate the action mode between drugs and targets. We find that most drugs have multiple organs support by inhibiting targets. And the drug-pathway analysis shows the characteristic of multitarget/pathways regulations, which result in several therapeutic modules, such as inflammation, contraction, and sustained angiogenesis. These results not only mirror the treatment feature of holistic medicine but also promote the development of new drugs. In addition, it points to the way for the treatment of complex diseases.

Complex diseases largely arise from an integration of genetic, environmental, and lifestyle factors. Yet, unlike the Mendelian diseases, which are caused by abnormalities to a single gene, complex diseases are chiefly unknown due to byzantine genetic and organic interactions. To treat chronic disease, there are several means in allopathic medicine such as cocktails of drugs and drug combination. But they are always weakly effective. In holistic medicine, the cardiovascular and cerebrovascular diseases were stratified into their distinct subtypes (CVDs and CBVDs) for an impedance match against proper drugs. Molecules with $\mathrm{BBB}$ permeability directly activate or block the targets located in brain and further boost the functional recovery after CBVDs by suppressing the complications, such as Alzheimer's disease and stroke pain. Compounds interact with nonbrain location targets such as sex hormone receptors that are effective in treating CVDs (hypertension, coronary artery disease, etc.).

As exemplified by CCDs, the present study demonstrates the effectiveness of holistic medicine in curing complex diseases from a molecular to system level and importantly provides a paradigm for the treatment of complex diseases in the near future.

\section{Conflict of Interests}

The authors declare that there is no conflict of interests regarding the publication of this paper.

\section{Acknowledgments}

The research is supported by the Fund of Northwest A\&F University, the National Natural Science Foundation of China (Grant nos. 31170796 and 81373892), and New Century Excellent Talents in University of Ministry of Education of China and is financially supported by China Postdoctoral Science Foundation (Grant no. 131891).

\section{References}

[1] S. Ventegodt, N. J. Andersen, and J. Merrick, "Holistic medicine: scientific challenges," TheScientificWorldJOURNAL, vol. 3, pp. 1108-1116, 2003.

[2] Y. Wang and A. Xu, "Zheng: a systems biology approach to diagnosis and treatments," Science, vol. 346, no. 6216, supplement, pp. S13-S15, 2014.

[3] W. M. Gesler, "Therapeutic landscapes: medical issues in light of the new cultural geography," Social Science \& Medicine, vol. 34, no. 7, pp. 735-746, 1992.

[4] C. Auffray, Z. Chen, and L. Hood, "Systems medicine: the future of medical genomics and healthcare," Genome Medicine, vol. 1, no. 1, article 2, 2009.

[5] J. Craig, "Complex diseases: research and applications," Nature Education, vol. 1, no. 1, p. 184, 2008.

[6] C. Ronco, M. Haapio, A. A. House, N. Anavekar, and R. Bellomo, "Cardiorenal syndrome," Journal of the American College of Cardiology, vol. 52, no. 19, pp. 1527-1539, 2008.

[7] X. Li, X. Xu, J. Wang et al., "A system-level investigation into the mechanisms of chinese traditional medicine: compound danshen formula for cardiovascular disease treatment," PLoS ONE, vol. 7, no. 9, Article ID e43918, 2012.

[8] P. Jiang, R. Liu, S. Dou et al., "Analysis of the constituents in rat plasma after oral administration of Shexiang Baoxin pill by HPLC-ESI-MS/MS," Biomedical Chromatography, vol. 23, no. 12, pp. 1333-1343, 2009.

[9] W. Tao, X. Xu, X. Wang et al., "Network pharmacology-based prediction of the active ingredients and potential targets of Chinese herbal Radix Curcumae formula for application to cardiovascular disease," Journal of Ethnopharmacology, vol. 145, no. 1, pp. 1-10, 2013. 
[10] X. Wang, X. Xu, Y. Li et al., "Systems pharmacology uncovers Janus functions of botanical drugs: activation of host defense system and inhibition of influenza virus replication," Integrative Biology, vol. 5, no. 2, pp. 351-371, 2013.

[11] H. Liu, J. Wang, W. Zhou, Y. Wang, and L. Yang, "Systems approaches and polypharmacology for drug discovery from herbal medicines: an example using licorice," Journal of Ethnopharmacology, vol. 146, no. 3, pp. 773-793, 2013.

[12] D. K. W. Mok and F.-T. Chau, "Chemical information of Chinese medicines: a challenge to chemist," Chemometrics and Intelligent Laboratory Systems, vol. 82, no. 1-2, pp. 210-217, 2006.

[13] T. W. Lau, F. F. Y. Lam, K. M. Lau et al., "Pharmacological investigation on the wound healing effects of Radix Rehmanniae in an animal model of diabetic foot ulcer," Journal of Ethnopharmacology, vol. 123, no. 1, pp. 155-162, 2009.

[14] H. Yu, J. Chen, X. Xu et al., "A systematic prediction of multiple drug-target interactions from chemical, genomic, and pharmacological data," PLoS ONE, vol. 7, no. 5, Article ID e37608, 2012.

[15] P. A. Heidenreich, J. G. Trogdon, O. A. Khavjou et al., "Forecasting the future of cardiovascular disease in the United States: a policy statement from the American Heart Association," Circulation, vol. 123, no. 8, pp. 933-944, 2011.

[16] M. V. S. Varma, R. S. Obach, C. Rotter et al., "Physicochemical space for optimum oral bioavailability: contribution of human intestinal absorption and first-pass elimination," Journal of Medicinal Chemistry, vol. 53, no. 3, pp. 1098-1108, 2010.

[17] X. Xu, W. Zhang, C. Huang et al., "A novel chemometric method for the prediction of human oral bioavailability," International Journal of Molecular Sciences, vol. 13, no. 6, pp. 6964-6982, 2012.

[18] P. Geladi and B. R. Kowalski, "Partial least-squares regression: a tutorial," Analytica Chimica Acta, vol. 185, pp. 1-17, 1986.

[19] T. Hou and X. Xu, "ADME evaluation in drug discovery," Molecular Modeling Annual, vol. 8, no. 12, pp. 337-349, 2002.

[20] A. Guerra, N. E. Campillo, and J. A. Páez, "Neural computational prediction of oral drug absorption based on CODES 2D descriptors," European Journal of Medicinal Chemistry, vol. 45, no. 3, pp. 930-940, 2010.

[21] T. L. Moda and A. D. Andricopulo, "Consensus hologram QSAR modeling for the prediction of human intestinal absorption," Bioorganic \& Medicinal Chemistry Letters, vol. 22, no. 8, pp. 2889-2893, 2012.

[22] Y. Yao, X. Zhang, Z. Wang et al., "Deciphering the combination principles of Traditional Chinese Medicine from a systems pharmacology perspective based on Ma-huang Decoction," Journal of Ethnopharmacology, vol. 150, no. 2, pp. 619-638, 2013.

[23] Y. Yamanishi, M. Kotera, M. Kanehisa, and S. Goto, "Drugtarget interaction prediction from chemical, genomic and pharmacological data in an integrated framework," Bioinformatics, vol. 26, no. 12, Article ID btq176, pp. i246-i254, 2010.

[24] D. S. Wishart, C. Knox, A. C. Guo et al., "DrugBank: a comprehensive resource for in silico drug discovery and exploration," Nucleic Acids Research, vol. 34, pp. D668-D672, 2006.

[25] L. Li, Y. Li, Y. Wang, and S. Zhang, "Prediction of BBB permeation based on molecular indices," Chinese Journal of Medicinal Chemistry, vol. 17, no. 4, pp. 221-228, 2007.

[26] R. B. Altman, "PharmGKB: a logical home for knowledge relating genotype to drug response phenotype," Nature Genetics, vol. 39, no. 4, article 426, 2007.

[27] X. Chen, Z. L. Ji, and Y. Z. Chen, “TTD: therapeutic target database," Nucleic Acids Research, vol. 30, no. 1, pp. 412-415, 2002.
[28] A. P. Davis, C. G. Murphy, R. Johnson et al., "The comparative toxicogenomics database: update 2013," Nucleic Acids Research, vol. 41, no. 1, pp. D1104-D1114, 2013.

[29] M. E. Smoot, K. Ono, J. Ruscheinski, P.-L. Wang, and T. Ideker, "Cytoscape 2.8: new features for data integration and network visualization," Bioinformatics, vol. 27, no. 3, Article ID btq675, pp. 431-432, 2011.

[30] C. Huang, C. Zheng, Y. Li, Y. Wang, A. Lu, and L. Yang, "Systems pharmacology in drug discovery and therapeutic insight for herbal medicines," Briefings in Bioinformatics, vol. 15, no. 5, pp. 710-733, 2014.

[31] G. Bindea, B. Mlecnik, H. Hackl et al., "ClueGO: a Cytoscape plug-in to decipher functionally grouped gene ontology and pathway annotation networks," Bioinformatics, vol. 25, no. 8, pp. 1091-1093, 2009.

[32] P. Knekt, J. Kumpulainen, R. Järvinen et al., "Flavonoid intake and risk of chronic diseases," The American Journal of Clinical Nutrition, vol. 76, no. 3, pp. 560-568, 2002.

[33] S. Egert, A. Bosy-Westphal, J. Seiberl et al., "Quercetin reduces systolic blood pressure and plasma oxidised lowdensity lipoprotein concentrations in overweight subjects with a high-cardiovascular disease risk phenotype: a double-blinded, placebo-controlled cross-over study," British Journal of Nutrition, vol. 102, no. 7, pp. 1065-1074, 2009.

[34] H.-J. Kwon, Y. B. Ryu, H. J. Jeong et al., "Rhodiosin, an antioxidant flavonol glycoside from Rhodiola rosea," Journal of the Korean Society for Applied Biological Chemistry, vol. 52, no. 5, pp. 486-492, 2009.

[35] B. J. Willcox, J. D. Curb, and B. L. Rodriguez, "Antioxidants in cardiovascular health and disease: key lessons from epidemiologic studies," The American Journal of Cardiology, vol. 101, no. 10, pp. S75-S86, 2008.

[36] C. R. Cederroth and S. Nef, "Soy, phytoestrogens and metabolism: a review," Molecular and Cellular Endocrinology, vol. 304, no. 1-2, pp. 30-42, 2009.

[37] N. I. Krinsky, "Carotenoids as antioxidants," Nutrition, vol. 17, no. 10, pp. 815-817, 2001.

[38] Y. Yamamoto and E. Oue, "Antihypertensive effect of quercetin in rats fed with a high-fat high-sucrose diet," Bioscience, Biotechnology and Biochemistry, vol. 70, no. 4, pp. 933-939, 2006.

[39] I. Mackraj, T. Govender, and S. Ramesar, "The antihypertensive effects of quercetin in a salt-sensitive model of hypertension," Journal of Cardiovascular Pharmacology, vol. 51, no. 3, pp. 239245, 2008.

[40] M.-X. Jiang, X.-F. Ruan, and Y. Xu, "Effects of Kanlijian on exercise tolerance, quality of life, and frequency of heart failure aggravation in patients with chronic heart failure," Chinese Journal of Integrative Medicine, vol. 12, no. 2, pp. 94-100, 2006.

[41] D. L. Tribble, "Further evidence of the cardiovascular benefits of diets enriched in carotenoids," The American Journal of Clinical Nutrition, vol. 68, no. 3, pp. 521-522, 1998.

[42] H. K. Kim, H. R. Park, J. S. Lee, T. S. Chung, H. Y. Chung, and J. Chung, "Down-regulation of iNOS and TNF- $\alpha$ expression by kaempferol via NF- $\kappa$ B inactivation in aged rat gingival tissues," Biogerontology, vol. 8, no. 4, pp. 399-408, 2007.

[43] G. L. Firestone and S. N. Sundar, "Anticancer activities of artemisinin and its bioactive derivatives," Expert Reviews in Molecular Medicine, vol. 11, article e32, 2009.

[44] J. B. Toledo, S. E. Arnold, K. Raible et al., "Contribution of cerebrovascular disease in autopsy confirmed neurodegenerative disease cases in the National Alzheimer's Coordinating Centre," Brain, vol. 136, no. 9, pp. 2697-2706, 2013. 
[45] A. R. Inácio, K. Ruscher, L. Leng, R. Bucala, and T. Deierborg, "Macrophage migration inhibitory factor promotes cell death and aggravates neurologic deficits after experimental stroke," Journal of Cerebral Blood Flow \& Metabolism, vol. 31, no. 4, pp. 1093-1106, 2011.

[46] A. Bierhaus, T. Fleming, S. Stoyanov et al., "Methylglyoxal modification of Nav 1.8 facilitates nociceptive neuron firing and causes hyperalgesia in diabetic neuropathy," Nature Medicine, vol. 18, no. 6, pp. 926-933, 2012.

[47] M. J. Cipolla, Q. Huang, and J. G. Sweet, "Inhibition of protein kinase $\mathrm{C} \beta$ reverses increased blood-brain barrier permeability during hyperglycemic stroke and prevents edema formation in vivo," Stroke, vol. 42, no. 11, pp. 3252-3257, 2011.

[48] M. Mendioroz, I. Fernández-Cadenas, A. Rosell et al., "Osteopontin predicts long-term functional outcome among ischemic stroke patients," Journal of Neurology, vol. 258, no. 3, pp. 486493, 2011.

[49] A. Pollak, A. Rokach, A. Blumenfeld, L. J. Rosen, L. Resnik, and R. D. Pollak, "Association of oestrogen receptor alpha gene polymorphism with the angiographic extent of coronary artery disease," European Heart Journal, vol. 25, no. 3, pp. 240-245, 2004.

[50] T. A. Kunnas, P. Laippala, A. Penttilä, T. Lehtimäki, and P. J. Karhunen, "Association of polymorphism of human $\alpha$ oestrogen receptor gene with coronary artery disease in men: a necropsy study," The British Medical Journal, vol. 321, no. 7256, pp. 273-274, 2000.

[51] K. M. Fischer, C. T. Cottage, M. H. Konstandin, M. Völkers, M. Khan, and M. A. Sussman, "Pim-1 kinase inhibits pathological injury by promoting cardioprotective signaling," Journal of Molecular and Cellular Cardiology, vol. 51, no. 4, pp. 554-558, 2011.

[52] M. van der Paardt, J. B. A. Crusius, M. A. García-González, B. A. C. Dijkmans, A. S. Peña, and I. E. van der Horst-Bruinsma, "Susceptibility to ankylosing spondylitis: no evidence for the involvement of transforming growth factor $\beta 1$ (TGFB1) gene polymorphisms," Annals of the Rheumatic Diseases, vol. 64, no. 4, pp. 616-619, 2005.

[53] G. Z. Feuerstein, T. Liu, and F. C. Barone, "Cytokines, inflammation, and brain injury: role of tumor necrosis factor-alpha," Cerebrovascular and Brain Metabolism Reviews, vol. 6, no. 4, pp. 341-360, 1994.

[54] J. C. Braz, K. Gregory, A. Pathak et al., "PKC- $\alpha$ regulates cardiac contractility and propensity toward heart failure," Nature Medicine, vol. 10, no. 3, pp. 248-254, 2004.

[55] S. A. Crone, Y.-Y. Zhao, L. Fan et al., "ErbB2 is essential in the prevention of dilated cardiomyopathy," Nature Medicine, vol. 8, no. 5, pp. 459-465, 2002.

[56] L. Maier, D. Bers, and J. Brown, "Calmodulin and $\mathrm{Ca}^{2+} /$ calmodulin kinases in the heart-physiology and pathophysiology," Cardiovascular Research, vol. 73, no. 4, pp. 629-630, 2007.

[57] S. Gately and W. W. Li, "Multiple roles of COX-2 in tumor angiogenesis: a target for antiangiogenic therapy," Seminars in Oncology, vol. 31, no. 7, pp. 2-11, 2004.

[58] T. Murohara and T. Asahara, "Nitric oxide and angiogenesis in cardiovascular disease," Antioxidants and Redox Signaling, vol. 4, no. 5, pp. 825-831, 2002.

[59] A. J. Marrogi, W. D. Travis, J. A. Welsh et al., "Nitric oxide synthase, cyclooxygenase 2, and vascular endothelial growth factor in the angiogenesis of non-small cell lung carcinoma," Clinical Cancer Research, vol. 6, no. 12, pp. 4739-4744, 2000.
[60] R. Aebersold and M. Mann, "Mass spectrometry-based proteomics," Nature, vol. 422, no. 6928, pp. 198-207, 2003.

[61] M. S. Joshi, C. Mineo, P. W. Shaul, and J. A. Bauer, "Biochemical consequences of the NOS3 Glu298Asp variation in human endothelium: altered caveolar localization and impaired response to shear," The FASEB Journal, vol. 21, no. 11, pp. 26552663, 2007.

[62] J.-F. Arnal, A.-T. Dinh-Xuan, M. Pueyo, B. Darblade, and J. Rami, "Endothelium-derived nitric oxide and vascular physiology and pathology," Cellular and Molecular Life Sciences, vol. 55, no. 8-9, pp. 1078-1087, 1999.

[63] C. Monaco, N. Terrando, and K. S. Midwood, “Toll-like receptor signaling: common pathways that drive cardiovascular disease and rheumatoid arthritis," Arthritis Care \& Research, vol. 63, no. 4, pp. 500-511, 2011.

[64] D. J. H. McCabe, P. Harrison, I. J. Mackie et al., "Platelet degranulation and monocyte-platelet complex formation are increased in the acute and convalescent phases after ischaemic stroke or transient ischaemic attack," British Journal of Haematology, vol. 125, no. 6, pp. 777-787, 2004.

[65] P. A. Cahill and E. M. Redmond, "Alcohol and cardiovascular disease-modulation of vascular cell function," Nutrients, vol. 4 , no. 4, pp. 297-318, 2012.

[66] L. Ferini-Strambi, A. S. Walters, and D. Sica, “The relationship among restless legs syndrome (Willis-Ekbom Disease), hypertension, cardiovascular disease, and cerebrovascular disease," Journal of Neurology, vol. 261, no. 6, pp. 1051-1068, 2014.

[67] J.-C. Reil, F. Custodis, K. Swedberg et al., "Heart rate reduction in cardiovascular disease and therapy," Clinical Research in Cardiology, vol. 100, no. 1, pp. 11-19, 2011.

[68] C. A. Herzog, R. W. Asinger, A. K. Berger et al., "Cardiovascular disease in chronic kidney disease. A clinical update from kidney disease: improving global outcomes (KDIGO)," Kidney International, vol. 80, no. 6, pp. 572-586, 2011.

[69] R. M. Freedom, F. S. Rosen, and A. S. Nadas, "Congenital cardiovascular disease and anomalies of the third and fourth pharyngeal pouch," Circulation, vol. 46, no. 1, pp. 165-172, 1972.

[70] L. Hood and M. Flores, "A personal view on systems medicine and the emergence of proactive $\mathrm{P} 4$ medicine: predictive, preventive, personalized and participatory," New Biotechnology, vol. 29, no. 6, pp. 613-624, 2012. 


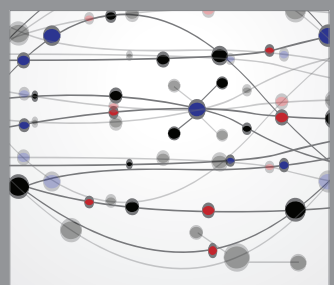

The Scientific World Journal
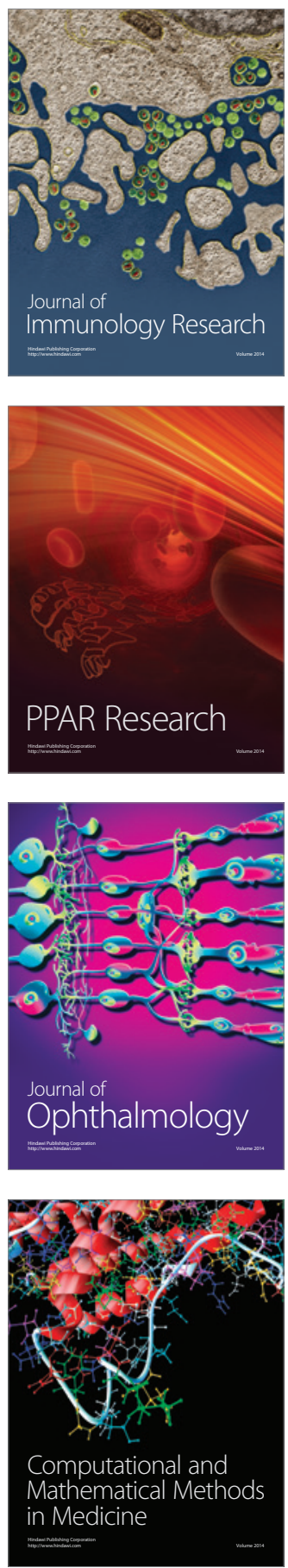

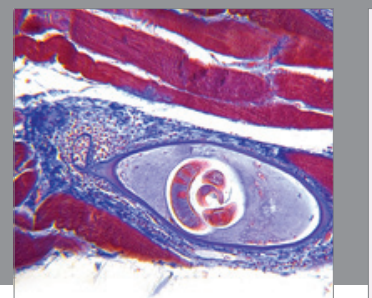

Gastroenterology

Research and Practice
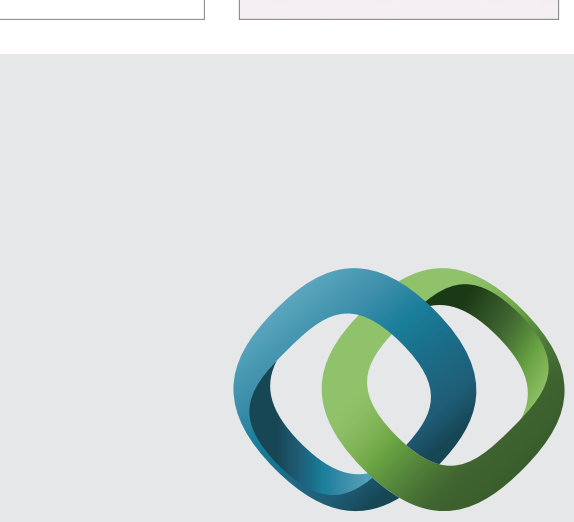

\section{Hindawi}

Submit your manuscripts at

http://www.hindawi.com
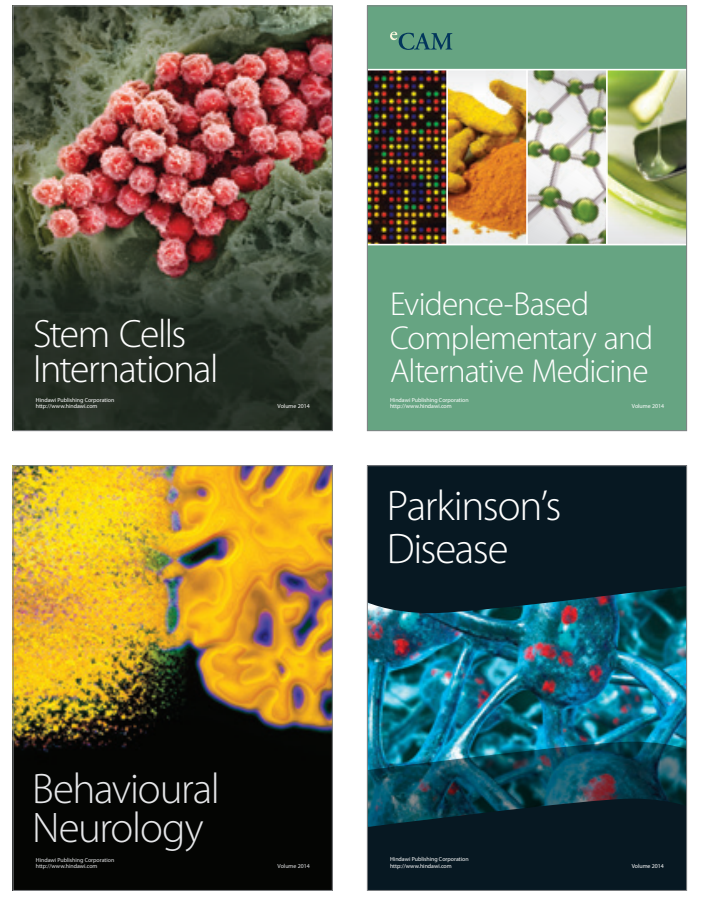
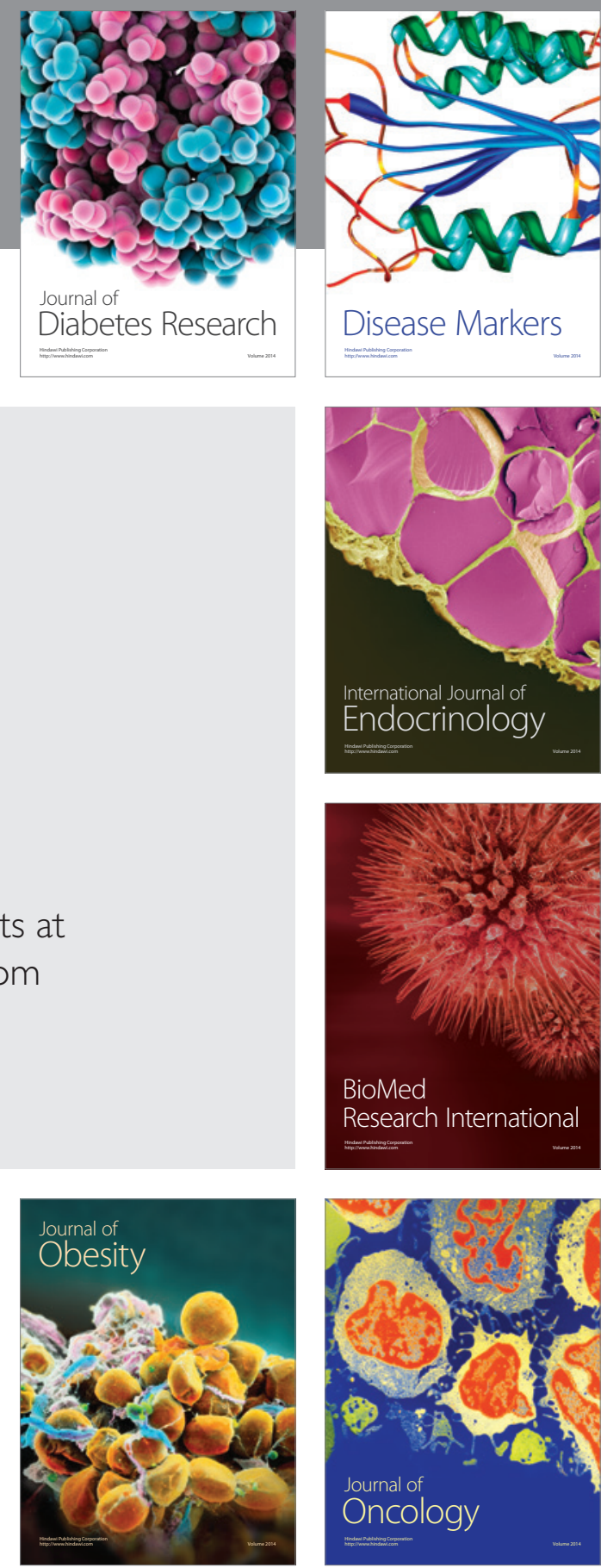

Disease Markers
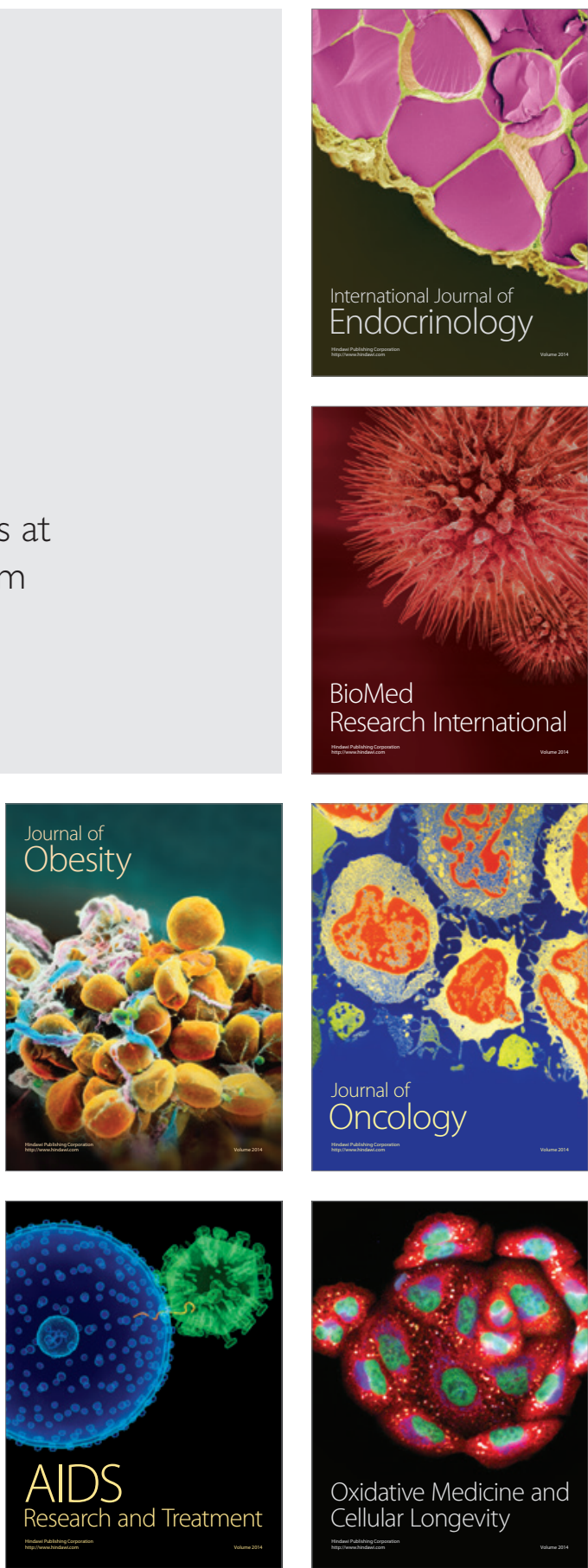